\title{
ADAPTIVE MULTIRESOLUTION DISCONTINUOUS GALERKIN SCHEMES FOR CONSERVATION LAWS
}

\author{
NUNE HOVHANNISYAN, SIEGFRIED MÜLLER, AND ROLAND SCHÄFER
}

\begin{abstract}
A multiresolution-based adaptation concept is proposed that aims at accelerating discontinuous Galerkin schemes applied to non-linear hyperbolic conservation laws. Opposite to standard adaptation concepts no error estimates are needed to tag mesh elements for refinement. Instead of this, a multiresolution analysis is performed on a hierarchy of nested grids for the data given on a uniformly refined mesh. This provides difference information between successive refinement levels that may become negligibly small in regions where the solution is locally smooth. Applying hard thresholding the data are highly compressed and local grid adaptation is triggered by the remaining significant coefficients. A central mathematical problem addressed in this work is then to show at least for scalar one-dimensional problems that choosing an appropriate threshold value, the adaptive solution is of the same accuracy as the reference solution on a uniformly refined mesh. Numerical comparisons demonstrate the efficiency of the concept.
\end{abstract}

\section{INTRODUCTION}

The solution of a non-linear hyperbolic conservation law typically develops discontinuities. In order to avoid instabilities in the numerical discretization, Finite Volume (FV) schemes are frequently used. These have been developed and investigated for many decades. An overview can be found in the textbooks of Godlewski and Raviart [22, Kröner [31, and LeVeque [33]. Although these schemes have proven to be very robust in practice, they suffer from discretization stencils that are growing with increasing order. In particular, on unstructured meshes this turns out to be a severe drawback. This problem can be overcome by using Discontinuous Galerkin (DG) schemes which are locally conservative, stable and high-order accurate. These methods can easily handle complex geometries and meshes with hanging nodes as well as approximations with a locally changing polynomial degree in different elements. In particular, they are easy to implement and allow for an efficient parallelization. After their introduction in 1973 by Reed and Hill [40, a major development was carried out by Cockburn et al. 12, 11, 9, 13, 14. A review on Runge-Kutta DG schemes for convection-dominated problems can be found in [15. By now DG methods have been applied to both ordinary and partial differential equations [10.

Received by the editor September 13, 2010 and, in revised form, October 20, 2011, March 6, 2012, and June 19, 2012.

2010 Mathematics Subject Classification. Primary 35L65, 65M12, 65M60, 65T60, 74S05.

This work has been performed with funding by the Deutsche Forschungsgemeinschaft in the Collaborative Research Center SFB 401 "Flow Modulation and Fluid-Structure Interaction at Airplane Wings" of RWTH Aachen University, Germany. 
Due to the formation of discontinuities the solution of a non-linear hyperbolic conservation law often locally exhibits steep gradients and large regions where it is smooth. To account for the highly non-uniform spatial behavior, we need numerical schemes that adequately resolve the different scales, i.e., use a high resolution only near sharp transition regions and singularities but a moderate resolution in regions with a smooth, slowly varying behavior of the solution. For this purpose, strategies for DG discretizations have been discussed or are under current investigation that aim at adapting the internal degrees of freedom, such as the spatial and temporal discretization or the discretization order, to the local behavior of the flow field. So far numerous refinement indicators have been developed that are based on interpolation error estimates of some key quantity using a priori knowledge of the solution. Although these concepts turn out to be very efficient in practice, they offer no reliable error control. For this purpose, error estimates are needed which provide a control of the actual error in the approximate solution. Bey and Oden [5] obtained a priori as well as a posteriori error estimates for adaptive strategies. By now many types of a posteriori error estimates have been designed to control the adaptive process. A lot of work has been done by Flaherty et al. 11, 41, Houston et al. [26, 25, 27, Dedner et al. 21], and recently Mavriplis et al. [46].

All these approaches have in common that they aim at estimating the error of the solution. However, in general there are no mathematical rigorous a priori or a posteriori error estimates available for non-linear systems of conservation laws such as Euler equations, MHD equations, and shallow water equations, neither for finite volume schemes nor finite element discretizations. Therefore we propose an alternative adaptation strategy that does not rely on the existence of some error estimator. The rationale behind its design is to accelerate a given DG scheme or a FV scheme (reference scheme) on a uniformly refined mesh (reference mesh) through computing actually only on a locally refined adapted subgrid, while preserving (up to a fixed constant multiple) the accuracy of the discretization on the full uniform grid. For this purpose, a multiresolution analysis (MRA) is performed, where the data corresponding to the current solution are represented as data on some coarse level and the fine scale information is encoded as arrays of detail coefficients of ascending resolution. The new data format reveals insight into the local behavior of the solution. It can be shown that the details become small with increasing refinement level when the underlying function is smooth. As suggested by this so-called cancellation property, we may determine a locally refined grid performing data compression on the array of detail coefficients using hard thresholding. This significantly reduces the complexity of the data. Based on the thresholded array local grid adaptation is performed, where we refine an element whenever there exists a significant detail. Of course, the crux in this context is to arrange this procedure in such a way that at no stage of the computation is there ever made use of the fully refined mesh.

Such multiresolution-based mesh adaptation methods using biorthogonal wavelets [17] have been introduced in [35, 18, and have been quite successful with FV solvers for compressible fluid flow; see Bramkamp et al. [6]. A comprehensive review on their development can be found in [36, and [19] provides some overview on recent trends. Note that the rate of decay of the details fastens with the increasing number of vanishing moments of the wavelets, i.e., more details may be discarded in smooth regions and the adaptive mesh becomes coarser. However, 
for biorthogonal wavelets to realize more vanishing moments requires that they extend the support of the wavelet functions. Its construction, in particular, becomes even more complicated on unstructured grid hierarchies. Therefore it is natural to extend the multiresolution-based mesh adaptation concept to higher order DG discretizations using so-called multiwavelets [44, 30]. Multiwavelets allow for higher order vanishing moments, while being supported on a single mesh element.

Objective. In the present work, we develop the concept of multiresolutionbased DG (MR-DG) schemes, where for the sake of analysis we focus on scalar one-dimensional conservation laws. We emphasize that none of the conceptual ingredients are restricted to this setting but can be extended straightforwardly to multidimensional systems of conservation laws. The key idea is to apply data compression by means of an MRA and hard thresholding. Instead of performing time evolution on the full set of equations on the uniform reference grid, we only evolve the equations of a reduced set of significant multiscale coefficients corresponding to a locally refined grid. Then we may introduce the perturbation error as the difference of the results obtained by performing computations with the reference scheme and the adaptive scheme. From a mathematical point of view it is of interest whether the perturbation error can be estimated by the threshold value. For scalar conservation laws we verify an a priori error estimate of the perturbation error; see Theorem 1. Since error estimates for the discretization error of the reference DG scheme are available in this case, cf. [12, the threshold value can be chosen such that the perturbation error and the discretization error are balanced. Due to the lack of similar error estimates for general systems of conservation laws, this choice may motivate the selection of a reasonable threshold value for practical problems. Recently, this was investigated numerically in [29] by performing parameter studies in case of the Euler equations.

Outline. In Section 2 we present the reference Runge-Kutta DG scheme that will provide us with data on a uniform reference mesh. In Section 3 we then summarize the key ingredients of the MRA based on Alpert's orthogonal multiwavelets that will allow for data compression of these data. In Section 4, we then derive the adaptive MR-DG scheme and give the basic algorithms. In order to analyze the perturbation error of the adaptive scheme we present in Section 5 a general framework. In particular, we will derive sufficient conditions that will ensure a priori estimates of the perturbation error. In Section 6 we verify these conditions to hold for our adaptive MR-DG scheme. Finally we have implemented the MR-DG scheme for scalar one-dimensional conservation laws. Numerical results in Section 7 demonstrate the efficiency of our concept. In particular, we discuss the choice of an "optimal" threshold value.

\section{The Reference DG SCheme}

Governing equations. In order to simplify the notation, we confine ourselves to one spatial dimension. We like to point out once more that the concepts extend to higher-dimensional problems as well. In the framework of adaptive MR-FV schemes this has been realized for the multidimensional case and has been successfully applied to complex configurations in fluid dynamics; cf. [6]. Here we consider the initial value problem for a scalar inhomogeneous conservation law

$$
\begin{aligned}
& u_{t}(t, x)+(f(u(t, x)))_{x}=s(u(t, x)), \quad t>0, \\
& u(0, x)=u_{0}(x), \quad x \in \mathbb{R} .
\end{aligned}
$$


If the initial data $u_{0} \in B V(\mathbb{R}) \cap L^{\infty}(\mathbb{R}) \cap L^{1}(\mathbb{R})$, the flux $f \in C^{1}(\mathbb{R})$ and the source term $s: \mathbb{R} \rightarrow \mathbb{R}$ satisfies a global Lipschitz condition with Lipschitz constant $L_{s}$ and $s(0)=0$, then there exists a unique entropy solution $u \in L^{\infty}\left(0, T, L_{\text {loc }}^{1}(\mathbb{R})\right)$ for all $T>0$; see 38,32 , 45. We will assume additionally that $u_{0}$ is compactly supported.

Weak formulation. The entropy solution is approximated by a DG scheme. Since computations are only performed on bounded domains and convergence analysis is only performed for compact sets, we confine ourselves to uniform finite discretizations of $\Omega:=[a, b]=\bigcup_{k \in \mathcal{I}} V_{k}$, where the cells $V_{k}:=\left[x_{k}, x_{k+1}\right], k \in \mathcal{I}:=$ $\{0, \ldots, N-1\}$, are determined by the discretization points $x_{k}:=a+k h$ with uniform spatial discretization $h=(b-a) / N$.

On this discretization we introduce the DG space $S^{p}:=\left\{w \in L^{2}([a, b]):\left.w\right|_{V_{k}} \in\right.$ $\left.\Pi_{p-1} \forall k \in \mathcal{I}\right\}$ of piecewise polynomial functions of degree less than $p$. For this finite-dimensional space we introduce two sets of basis functions, $\Phi:=\left\{\varphi_{k, i}\right\}_{k \in \mathcal{I}, i \in \mathcal{P}}$ and $\tilde{\Phi}:=\left\{\tilde{\varphi}_{k, i}\right\}_{k \in \mathcal{I}, i \in \mathcal{P}}$ with $\mathcal{P}:=\{0, \ldots, p-1\}$ such that $S^{p}=\operatorname{span} \Phi=\operatorname{span} \tilde{\Phi}$. We assume that these functions fulfill the biorthogonality relation

$$
\left\langle\varphi_{k^{\prime}, i^{\prime}}, \tilde{\varphi}_{k, i}\right\rangle_{\Omega}=\delta_{i, i^{\prime}} \delta_{k, k^{\prime}}
$$

and are compactly supported, i.e., $\operatorname{supp} \varphi_{k, i}=\operatorname{supp} \tilde{\varphi}_{k, i}=V_{k}, i \in \mathcal{P}$. Here $\langle f, g\rangle_{\Omega}:=\int_{\Omega} f(x) g(x) d x$ denotes the standard $L^{2}$-inner product. A typical example are the shifts and translates of the Legendre polynomials normalized with respect to $L^{\infty}$ and $L^{1}$, respectively. In order to derive a discretization of the initial value problem (2.1), we now assume that the approximate solution can be written as an expansion of the basis $\Phi$, i.e.,

$$
u_{h}(t, \cdot)=\sum_{k \in \mathcal{I}} \sum_{i \in \mathcal{P}} v_{k, i}(t) \varphi_{k, i}(\cdot) \in S^{p} .
$$

The coefficients $v_{k, i}$ are determined by the biorthogonality relation (2.2) as

$$
v_{k, i}(t)=\left\langle u_{h}(t, \cdot), \tilde{\varphi}_{k, i}\right\rangle_{\Omega} .
$$

In order to derive an evolution equation for these coefficients we rewrite (2.1) in a weak formulation, where we multiply (2.1) by $\tilde{\varphi}_{k, i}$ and integrate over its support $V_{k}$. Inserting (2.3) and performing integration by parts we end up with

$$
\frac{d}{d t} v_{k, i}(t)+\left.f\left(u_{h}(t, x)\right) \tilde{\varphi}_{k, i}(x)\right|_{x_{k}^{+}} ^{x_{k+1}^{-}}-\left\langle f\left(u_{h}\right), \tilde{\varphi}_{k, i}^{\prime}\right\rangle_{V_{k}}=\left\langle s\left(u_{h}\right), \tilde{\varphi}_{k, i}\right\rangle_{V_{k}},
$$

where we employ biorthogonality (2.2) and the local support of the basis functions. Here $x^{ \pm}$always denotes the right-sided $(+)$and left-sided $(-)$limit at position $x$, respectively.

Approximation. Since the basis functions and, hence, the approximate solution $u_{h}$ suffer from jumps at the interval points $x_{k}$, the flux evaluations $f\left(u_{h}\left(t, x^{ \pm}\right)\right)$ are replaced by numerical fluxes $F\left(u_{h}\left(t, x_{k}^{-}\right), u_{h}\left(t, x_{k}^{+}\right)\right)$, where $F: \mathbb{R}^{2} \rightarrow \mathbb{R}$ is a two-point flux function such that

(1) $F$ is consistent with the flux $f$, i.e., $F(v, v)=f(v)$ for all $v \in \mathbb{R}$,

(2) $F$ is Lipschitz continuous, i.e., $\left|F\left(u_{1}, v_{1}\right)-F\left(u_{2}, v_{2}\right)\right| \leq L_{1}\left|u_{1}-u_{2}\right|+L_{2} \mid v_{1}-$ $v_{2} \mid$ for all $\left|u_{i}\right|,\left|v_{i}\right| \leq C, i=1,2$, with $C>0$ arbitrary but fixed;

(3) $F$ is monotone, i.e., non-decreasing in the first argument and non-increasing in the second argument. 
Some typical examples are the flux functions due to Engquist-Osher, (local) LaxFriedrichs, Godunov, and Roe with entropy fix, cf. 22. In general, monotonicity only holds if a Courant-Friedrichs-Levy (CFL) condition is satisfied for the time and space discretization. Finally, we obtain the semi-discrete DG method

$$
\frac{d}{d t} v_{k, i}(t)+\left.F\left(u_{h}(t, x), u_{h}(t, x)\right) \tilde{\varphi}_{k, i}(x)\right|_{x_{k}^{+}} ^{x_{k+1}^{-}}-\left\langle f\left(u_{h}\right), \tilde{\varphi}_{k, i}^{\prime}\right\rangle_{V_{k}}=\left\langle s\left(u_{h}\right), \tilde{\varphi}_{k, i}\right\rangle_{V_{k}} .
$$

For a higher-order time discretization we apply $m$-stage total variation diminishing Runge-Kutta (TVD-RK) methods introduced by Shu and Osher [43. These fit into the class of strong stability preserving Runge-Kutta methods (SSP-RK); cf. 23. Characteristic of TVD-RK schemes is that each stage is as a convex combination of forward Euler discretizations. Introducing the time discretization $t_{n+1}:=t_{n}+\tau$, where for reasons of simplicity the time step $\tau>0$ is assumed to be constant, a single forward Euler step applied to the semi-discrete DG scheme (2.5) reads

$$
v_{k, i}^{n+1}=v_{k, i}^{n}-\tau\left(B_{k, i}^{n}-G_{k, i}^{n}-S_{k, i}^{n}\right),
$$

where the numerical flux $F_{k}^{n}$, the numerical flux balance $B_{k, i}^{n}$, the flux integral $G_{k, i}^{n}$, and the source integral $S_{k, i}^{n}$ are defined by

$$
\begin{aligned}
F_{k}^{n} & :=F\left(u_{h}\left(t^{n}, x_{k}^{-}\right), u_{h}\left(t^{n}, x_{k}^{+}\right)\right), \\
B_{k, i}^{n} & :=F_{k+1}^{n} \tilde{\varphi}_{k, i}\left(x_{k+1}^{-}\right)-F_{k}^{n} \tilde{\varphi}_{k, i}\left(x_{k}^{+}\right), \\
G_{k, i}^{n} & :=\left\langle f\left(u_{h}\left(t^{n}, \cdot\right)\right), \tilde{\varphi}_{k, i}^{\prime}\right\rangle_{V_{k}}, \quad S_{k, i}^{n}:=\left\langle s\left(u_{h}\left(t^{n}, \cdot\right)\right), \tilde{\varphi}_{k, i}\right\rangle_{V_{k}} .
\end{aligned}
$$

Limiting. In order to avoid oscillations at discontinuities that are characteristic for hyperbolic conservation laws, we apply a limiter to the coefficients of the DG discretization (2.6). Motivated by the van Leer limiter and the Shu limiter we use the following limiter that is independent of the polynomial degree $p$ of the ansatz space:

$$
\tilde{v}_{k, 0}:=v_{k, 0}, \quad \tilde{v}_{k, i}:=c_{k} v_{k, i}, i>0,
$$

with coefficient

$$
\begin{aligned}
c_{k}:=m(1, & \frac{v_{k, 0}-v_{k-1,0}}{\bar{v}_{k}^{+}}, \frac{v_{k+1,0}-v_{k, 0}}{\bar{v}_{k}^{+}}, \\
& \left.\frac{v_{k, 0}-v_{k-1,0}}{\bar{v}_{k}^{-}}, \frac{v_{k+1,0}-v_{k, 0}}{\bar{v}_{k}^{-}}, \frac{C_{\alpha} h^{\alpha-1}}{\max _{x \in V_{k}}\left|v^{\prime}(x)\right|}\right)
\end{aligned}
$$

and constants $C_{\alpha}>0$ and $\alpha>1$ defined by the standard minmod function

$$
m\left(a_{1}, \ldots, a_{n}\right):= \begin{cases}s \min _{1 \leq i \leq n}\left|a_{i}\right|, & \operatorname{sgn}\left(a_{1}\right)=\ldots=\operatorname{sgn}\left(a_{n}\right)=s, \\ 0, & \text { otherwise }\end{cases}
$$

and the contribution of the higher order coefficients to $u_{h}$ at the cell interfaces

$$
\bar{v}_{k}^{-}:=\sum_{i \in \mathcal{P}^{*}} v_{k, i} \varphi_{k, i}\left(x_{k+1}^{-}\right), \quad \bar{v}_{k}^{+}:=-\sum_{i \in \mathcal{P}^{*}} v_{k, i} \varphi_{k, i}\left(x_{k}^{+}\right), \quad \mathcal{P}^{*}:=\mathcal{P} \backslash\{0\} .
$$

A uniform notation for the effect of (2.10) on the data is the following: for the limited data $\tilde{\mathbf{v}}$ obtained from $\mathbf{v}$ we have

$$
\begin{gathered}
\tilde{v}\left(x_{k}^{+}\right)-v_{k, 0}=c_{k}^{+}\left(v\left(x_{k}^{+}\right)-v_{k, 0}\right), \\
\tilde{v}\left(x_{k+1}^{-}\right)-v_{k, 0}=c_{k}^{-}\left(v\left(x_{k+1}^{-}\right)-v_{k, 0}\right)
\end{gathered}
$$


with $c_{k}^{ \pm} \in[0,1]$. If the limiter does not change the data in cell $V_{k}$, then $c_{k}^{ \pm}=1$. This also holds for the van Leer and the Shu limiter. For the limiter (2.10) we, in particular, have $c_{k}^{+}=c_{k}^{-}=c_{k}$.

In 42 it is proven that the TVBM conditions in [12] hold with $\theta=1$ and thus the DG scheme converges towards a weak solution of (2.1). Moreover it is verified that the entropy enforcing conditions

$$
\begin{gathered}
\left|\tilde{v}_{k}^{ \pm}\right| \leq C_{\alpha} h^{\alpha} \\
\left|\tilde{v}(x)-\tilde{v}_{k, 0}\right|=\left|\sum_{i \in P^{*}} \tilde{v}_{k, i} \varphi_{k, i}(x)\right| \leq C_{\alpha} h^{\alpha-1} \quad \text { for all } x \in V_{k}
\end{gathered}
$$

hold. The functions $\tilde{v}_{k}^{ \pm}:=c_{k} \bar{v}_{k}^{ \pm}$are defined by (2.10a) and (2.10d) and correspond only to the higher order coefficients. In practice, the conditions (2.12) and (2.13) are usually not enforced, i.e., $C_{\alpha}$ is chosen very large. In the homogeneous case with convex flux it is known that (2.12) is not necessary to enforce convergence towards the entropy solution; see [39].

Convergence. Typically, convergence of (2.6) is investigated for the averaged function of piecewise constant data

$$
\hat{u}_{h}(t, x):=\left\langle u_{h}(t, \cdot), \tilde{\varphi}_{k, 0}\right\rangle_{V_{k}}=v_{k, 0}^{n}, \quad x \in V_{k}, t_{n} \leq t<t_{n+1},
$$

where we assume that $\tilde{\varphi}_{k, 0}=h^{-1} \chi_{V_{k}}$. Since the limiter (2.10) satisfies the TVBM conditions in [12] as well as the entropy enforcing conditions (2.12) and (2.13) in [8], convergence in the mean towards the entropy solution of (2.1) can be verified following the convergence proof in 12 for homogeneous problems. Since we assume that $s(0)=0$, the proof also applies to inhomogeneous problems.

\section{Multiresolution analysis, Data COMPREssion AND GRID adAptation}

The DG discretization typically works on an array of coefficients. In order to realize a certain target accuracy at the expense of a possibly low number of degrees of freedom, viz. a possibly low computational effort, one should keep the size of the cells large wherever the data exhibit little variation, reflecting a high regularity of the searched solution components. Our analysis of the local regularity behavior of the data is based on the concept of multiwavelets; cf. 30. This can be considered a natural extension of the MRA for cell averages corresponding to a FV discretization, i.e., $p=1$, where biorthogonal wavelets have been used to construct an appropriate MRA; cf. [20, 35]. Here we will briefly summarize the basic ideas of the MRA concept. Although we will confine ourselves to the one-dimensional case, the framework is applicable to arbitrary discretizations in higher dimensions. The core ingredients are (i) a hierarchy of nested grids, (ii) (biorthogonal) multiwavelets and (iii) the multiscale decomposition. Note that the multiwavelet MRA generalizes the discrete MRA in [4, 47] motivated by Harten's original discrete framework 24] for cell averages. Finally we conclude with the construction of a locally refined grid using the MRA and data compression.

Nested grid hierarchy. The starting point for the construction of an MRA is a sequence of nested grids. Here we confine ourselves to $1 \mathrm{D}$ dyadic grid refinements on the interval $\Omega=[a, b]$. Let $\mathcal{G}_{l}:=\left\{V_{l, k}\right\}_{k \in \mathcal{I}_{l}}, \mathcal{I}_{l}=\left\{0, \ldots, N_{l}-1\right\}, l \in \mathbb{N}_{0}$, be a sequence of grids with increasing resolution. These meshes are composed of the intervals $V_{l, k}=\left[x_{l, k}, x_{l, k+1}\right]$ determined by the discretization points $x_{l, k}:=a+k h_{l}$ 
with uniform spatial discretization $h_{l}=(b-a) / N_{l}$. The cell center is given by $\hat{x}_{l, k}=\left(x_{l, k}+x_{l, k+1}\right) / 2$. Obviously, the resulting grid hierarchy is nested, i.e.,

$$
V_{l, k}=V_{l+1,2 k} \cup V_{l+1,2 k+1}, \quad \forall k \in \mathcal{I}_{l}, l \in \mathbb{N}_{0} .
$$

Multiresolution analysis. On the set of grids described above we apply the concept of multiresolution analysis 34. We consider the set of scaling functions $\Phi_{l}^{p}:=\left\{\varphi_{l, k, i}: k \in \mathcal{I}_{l}, i \in \mathcal{P}\right\}$ of shifted and $L^{\infty}$-scaled Legendre polynomials. The set $\Phi_{l}^{p}$ forms a basis for

$$
S_{l}^{p}=\left\{f \in L^{2}([a, b]):\left.f\right|_{V_{l, k}} \in \Pi_{p-1} \forall k \in \mathcal{I}_{l}\right\}
$$

of piecewise polynomial functions of degree less than $p$. Multiwavelets $\Psi_{l}^{p}:=$ $\left\{\psi_{l, k, i}: k \in \mathcal{I}_{l}, i \in \mathcal{P}\right\}$ on level $\ell$ are then chosen so that they form a basis for the orthogonal complement space $S_{l+1}^{p} \backslash S_{l}^{p}$. The union of $\Phi_{l}^{p}$ and $\Psi_{l}^{p}$ forms a basis for $S_{l+1}^{p}$, i.e.,

$$
\operatorname{span}\left(\Phi_{\ell}\right) \oplus \operatorname{span}\left(\Psi_{\ell}\right)=S_{\ell+1}^{p} .
$$

Here we apply Alpert's multiwavelets [2, 3, which are piecewise polynomials and form an orthogonal system to the Legendre polynomials. Whereas wavelets are in general continuous functions, this regularity is lost when passing to Alpert's multiwavelets. By shifts and translates we then determine $\varphi_{l, k, i}$ and $\psi_{l, k, i}$ that are referred to as the primal scaling functions and primal wavelet functions, respectively. These are assumed to be normalized with respect to $L^{\infty}$. By $\tilde{\varphi}_{l, k, i}$ and $\tilde{\psi}_{l, k, i}$ we will denote the dual scaling functions and dual wavelet functions, respectively, defined as the $L^{1}$-scaled counterparts. Thus the functions of the primal and dual systems have the following properties that will be exploited in the analysis presented in subsequent sections.

Proposition 1 (Properties of multiresolution analysis). For the system of primal scaling functions and multiwavelets determined as shifts and translates of the Legendre polynomials and Alpert's multiwavelets as well as their dual counterparts, the following conditions hold true;

(i) The primal and dual scaling functions and wavelets are locally supported, i.e.,

$$
\operatorname{supp} \varphi_{l, k, i}=\operatorname{supp} \psi_{l, k, i}=\operatorname{supp} \tilde{\varphi}_{l, k, i}=\operatorname{supp} \tilde{\psi}_{l, k, i}=V_{l, k} .
$$

(ii) They are uniformly bounded with respect to $L^{\infty}$ and $L^{1}$, respectively, i.e.,

$$
\begin{aligned}
\left\|\varphi_{l, k, i}\right\|_{L^{\infty}([a, b])} \lesssim 1, \quad\left\|\psi_{l, k, i}\right\|_{L^{\infty}([a, b])} \lesssim 1, \\
\left\|\tilde{\varphi}_{l, k, i}\right\|_{L^{1}([a, b])} \lesssim 1, \quad\left\|\tilde{\psi}_{l, k, i}\right\|_{L^{1}([a, b])} \lesssim 1,
\end{aligned}
$$

where "§" denotes a bound up to some constant independent of the level $l$ and the position $k$.

(iii) Each multiwavelet provides vanishing moments of order $M_{p, i}=p+i$, i.e.,

$$
\left\langle P, \tilde{\psi}_{l, k, i}\right\rangle_{[a, b]}=0, \quad \forall P \in \Pi_{M_{p, i}-1} .
$$

(iv) They are biorthogonal, i.e.,

$$
\begin{array}{ll}
\left\langle\varphi_{l, k, i}, \tilde{\varphi}_{l, k^{\prime}, i^{\prime}}\right\rangle_{[a, b]}=\delta_{i, i^{\prime}} \delta_{k, k^{\prime}}, & \left\langle\varphi_{l, k, i}, \tilde{\psi}_{l, k^{\prime}, i^{\prime}}\right\rangle_{[a, b]}=0, \\
\left\langle\psi_{l, k, i}, \tilde{\psi}_{l, k^{\prime}, i^{\prime}}\right\rangle_{[a, b]}=\delta_{i, i^{\prime}} \delta_{k, k^{\prime}}, & \left\langle\psi_{l, k, i}, \tilde{\varphi}_{l, k^{\prime}, i^{\prime}}\right\rangle_{[a, b]}=0 .
\end{array}
$$


(v) There exists the two-scale decomposition

$\tilde{\varphi}_{l, k, i}=\sum_{j \in \mathcal{P}} \sum_{s=0}^{1} \tilde{m}_{i, j}^{0, s} \tilde{\varphi}_{l+1,2 k+s, j}, \quad \tilde{\psi}_{l, k, i}=\sum_{j \in \mathcal{P}} \sum_{s=0}^{1} \tilde{m}_{i, j}^{1, s} \tilde{\varphi}_{l+1,2 k+s, j}, i \in \mathcal{P}$,

$\tilde{\varphi}_{l+1,2 k+s, i}=\sum_{j \in \mathcal{P}}\left(\tilde{g}_{i, j}^{0, s} \tilde{\varphi}_{l, k, j}+\tilde{g}_{i, j}^{1, s} \tilde{\psi}_{l, k, j}\right), s \in\{0,1\}, i \in \mathcal{P}$,

where the mask coefficients are determined as

$$
\begin{array}{rll}
\tilde{m}_{i, j}^{0, s} & =\left\langle\tilde{\varphi}_{l, k, i}, \varphi_{l+1,2 k+s, j}\right\rangle_{[a, b]}, & \tilde{m}_{i, j}^{1, s}=\left\langle\tilde{\psi}_{l, k, i}, \varphi_{l+1,2 k+s, j}\right\rangle_{[a, b]}, \\
\tilde{g}_{i, j}^{0, s} & =\left\langle\varphi_{l+1,2 k+s, i}, \tilde{\varphi}_{l, k, j}\right\rangle_{[a, b]}, & \tilde{g}_{i, j}^{1, s}=\left\langle\varphi_{l+1,2 k+s, i}, \tilde{\psi}_{l, k, j}\right\rangle_{[a, b]} .
\end{array}
$$

(vi) Any function $u \in L_{2}([a, b])$ can be approximated by the sequence of projections

$u_{L}=\sum_{k \in \mathcal{I}_{L}} \sum_{i \in \mathcal{P}} u_{L, k, i} \varphi_{L, k, i}=\sum_{k \in \mathcal{I}_{0}} \sum_{i \in \mathcal{P}} u_{0, k, i} \varphi_{0, k, i}+\sum_{l=0}^{L-1} \sum_{k \in \mathcal{I}_{l}} \sum_{i \in \mathcal{P}} d_{l, k, i} \psi_{l, k, i}$

that is converging to $u$ in $L^{2}$, where the single-scale coefficients and wavelet coefficients (details) in (3.12) are determined by

$$
u_{l, k, i}:=\left\langle u, \tilde{\varphi}_{l, k, i}\right\rangle_{[a, b]}, \quad d_{l, k, i}:=\left\langle u, \tilde{\psi}_{l, k, i}\right\rangle_{[a, b]} .
$$

(vii) For the coefficients there exists the two-scale decomposition

$$
\text { (a) } u_{l, k, i}=\sum_{j \in \mathcal{P}} \sum_{s=0}^{1} \tilde{m}_{i, j}^{0, s} u_{l+1,2 k+s, j}, \quad(b) d_{l, k, i}=\sum_{j \in \mathcal{P}} \sum_{s=0}^{1} \tilde{m}_{i, j}^{1, s} u_{l+1,2 k+s, j}, i \in \mathcal{P},
$$

$$
u_{l+1,2 k+s, i}=\sum_{j \in \mathcal{P}}\left(\tilde{g}_{i, j}^{0, s} u_{l, k, j}+\tilde{g}_{i, j}^{1, s} d_{l, k, j}\right), s \in\{0,1\}, i \in \mathcal{P},
$$

(viii) The dual scaling function $\tilde{\varphi}_{l, k, 0}$ coincides with the $L^{1}$-normalized characteristic function, i.e., $\tilde{\varphi}_{l, k, 0}=h_{l}^{-1} \chi_{V_{l, k}}$ and, hence, $u_{l, k, 0}$ coincides with the cell average of $u$ on $V_{l, k}$.

(ix) There exist constants $D_{r, i}$ such that

$$
\left\|\frac{d^{r}}{d x^{r}} \varphi_{l, k, i}\right\|_{L^{\infty}\left(V_{l, k}\right)} \leq h_{l}^{-r} D_{r, i} .
$$

Cancellation property. The vanishing moments (3.7) and the normalization (3.6) imply that the details become small with increasing refinement level when the underlying function is locally smooth, i.e.,

$$
\left|\left\langle u, \tilde{\psi}_{l, k, i}\right\rangle_{[a, b]}\right| \leq \inf _{P \in \Pi_{M-1}}\left|\left\langle u-P, \tilde{\psi}_{l, k, i}\right\rangle_{[a, b]}\right| \lesssim 2^{-l M}\|u\|_{W^{1, M}\left(V_{l, k}\right)}
$$

Here we assume that the grid hierarchy is quasi-uniform in the sense that the diameters of the cells on each level $l$ are proportional to $2^{-l}$. More precisely, the details decay at a rate of at least $2^{-l M}$, provided that the function $u$ has sufficient regularity on the support of the multiwavelet, i.e., $u \in W^{1, M}\left(V_{l, k}\right)$, and the multiwavelet has vanishing moments of order $M$. In fact, the higher the $M$ the more details may be discarded in smooth regions. Typically, the minimal number of vanishing moments is determined by $M=M_{p, i} \geq p$. Therefore, opposite to 
biorthogonal wavelets, the number of vanishing moments can easily be improved by increasing the order $p$ without enlarging the support.

Multi-scale transformation. In order to exploit the above compression potential, the idea is to transform the array of single-scale coefficients corresponding to a finest uniform discretization level

$$
\mathbf{u}_{L}:=\left(\mathbf{u}_{L, k}\right)_{k \in \mathcal{I}_{L}}, \quad \mathbf{u}_{L, k}:=\left(u_{L, k, i}\right)_{i \in \mathcal{P}}
$$

into a sequence of coarse grid data $\mathbf{u}_{0}$ and details $\mathbf{d}_{l}$,

$$
\mathbf{u}_{0}:=\left(\mathbf{u}_{0, k}\right)_{k \in \mathcal{I}_{0}}, \quad \mathbf{d}_{l}:=\left(\mathbf{d}_{l, k}\right)_{k \in \mathcal{I}_{l}}, \quad \mathbf{d}_{l, k}:=\left(d_{l, k, i}\right)_{i \in \mathcal{P}}, \quad l=0, \ldots, L-1,
$$

representing the successive update from a coarser resolution to a finer resolution. Here the coefficients $u_{l, k, i}$ and $d_{l, k, i}$ are defined according to (3.13). In fact, the single-scale and the multi-scale representations realize the change of basis in (3.12).

A successive application of the relations (3.14) decomposes the array $\mathbf{u}_{L}$ into coarse scale coefficients $\mathbf{u}_{0}$ and higher level fluctuations $\mathbf{d}_{0}, \ldots, \mathbf{d}_{L-1}$. We refer to this transformation as the multi-scale transformation determined by the multi-scale operator $\mathcal{M}_{L}: \mathbf{u}_{L} \longrightarrow\left(\mathbf{u}_{0}, \mathbf{d}_{0}, \ldots, \mathbf{d}_{L-1}\right)$. It is reversed by recursively applying the two-scale relation (3.15). The resulting inverse multi-scale transformation is described by the inverse multi-scale operator $\mathcal{M}_{L}^{-1}:\left(\mathbf{u}_{0}, \mathbf{d}_{0}, \ldots, \mathbf{d}_{L-1}\right) \longrightarrow \mathbf{u}_{L}$.

Thresholding and approximation. Due to the cancellation property, detail coefficients might become negligibly small whenever the underlying function is locally smooth. This gives rise to hard thresholding characterized by the index set

$$
\mathcal{D}_{\varepsilon}:=\left\{(l, k, i):\left|d_{l, k, i}\right|>\varepsilon_{l}\right\} .
$$

Here $\varepsilon \geq 0$ denotes the threshold value. The level-dependent threshold values $\varepsilon_{l}$ are determined by an appropriate scaling of $\varepsilon$. Later on we will choose $\varepsilon_{l}:=2^{l-L} \varepsilon$.

For an arbitrary index set $\mathcal{D}$ of multi-scale coefficients the threshold operator $\mathcal{T}_{\mathcal{D}}:\left(\mathbf{u}_{0}, \mathbf{d}_{0}, \ldots, \mathbf{d}_{L-1}\right) \longrightarrow\left(\tilde{\mathbf{u}}_{0}, \tilde{\mathbf{d}}_{0}, \ldots, \tilde{\mathbf{d}}_{L-1}\right)$ is defined element-wise by

$$
\tilde{d}_{l, k, i}:=\left\{\begin{array}{ll}
d_{l, k, i}, & (l, k, i) \in \mathcal{D} \\
0, & \text { otherwise }
\end{array}, \quad \tilde{u}_{0, k, i}:=u_{0, k, i}, \quad k \in \mathcal{I}_{0} .\right.
$$

Note that thresholding is not performed on the coarse-scale averages in order to maintain the conservation property of the adaptive DG scheme.

From this operator we define the approximation operator

$$
\mathcal{A}_{\mathcal{D}}:=\mathcal{M}_{L}^{-1} \mathcal{T}_{\mathcal{D}} \mathcal{M}_{L}
$$

and, in particular for $\mathcal{D}=\mathcal{D}_{\varepsilon}$,

$$
\mathcal{A}_{\varepsilon}:=\mathcal{M}_{L}^{-1} \mathcal{T}_{\mathcal{D}_{\varepsilon}} \mathcal{M}_{L}
$$

The error introduced by thresholding can be estimated proceeding in analogy to classical wavelet analysis, cf. [16], using the properties listed in Proposition 1 .

Proposition 2 (Approximation error). Let $\Omega=[a, b]$ be bounded and $\varepsilon_{l}=\bar{a}^{l-L} \varepsilon$ with $\bar{a}>1$. Then the approximation error with respect to the set of significant details $\mathcal{D}_{\varepsilon}$ is uniformly bounded with respect to $L^{q}(\Omega), q \in[1, \infty]$, i.e.,

$$
\left\|u_{L}-u_{L, \mathcal{D}_{\varepsilon}}\right\|_{L^{q}(\Omega)} \leq C_{t h r} \varepsilon \quad \text { and } \quad\left\|\hat{u}_{L}-\hat{u}_{L, \mathcal{D}_{\varepsilon}}\right\|_{L^{q}(\Omega)} \leq C_{t h r} \varepsilon,
$$

for some constant $C_{t h r}>0$ independent of $L, \varepsilon$. Here $u_{L}$ and $u_{L, \mathcal{D}_{\varepsilon}}$ are the projections according to (3.12) corresponding to the coefficients $\mathbf{u}_{L}$ and $\mathcal{A}_{\varepsilon} \mathbf{u}_{L}$. 
(a) single-scale

\begin{tabular}{|l|l|l|l|l|l|l|l|}
\hline $\mathbf{u}_{3,0}$ & $\mathbf{u}_{3,1}$ & $\mathbf{u}_{3,2}$ & $\mathbf{u}_{3,3}$ & $\mathbf{u}_{3,4}$ & $\mathbf{u}_{3,5}$ & $\mathbf{u}_{3,6}$ & $\mathbf{u}_{3,7}$ \\
\hline
\end{tabular}

(c) thresholding

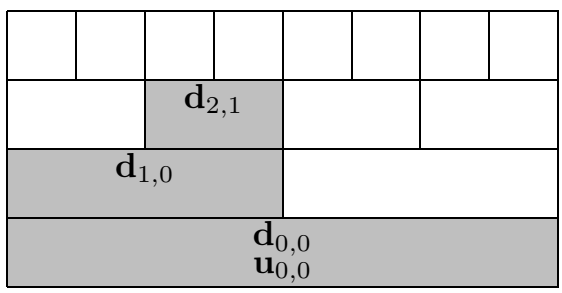

(b) multi-scale



(d) adaptive grid

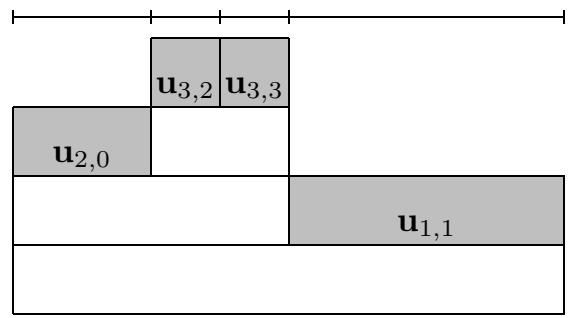

FiguRE 1. Multi-scale transformation (a), (b), thresholding (c) and the adaptive grid (d).

A detailed proof can be found in [42, p. $51 \mathrm{ff}$.

Grid adaptation. From the index set $\mathcal{D}$ we now determine a locally refined grid. This will be characterized by an index set $\mathcal{G} \subset\left\{(l, k): k \in \mathcal{I}_{l}, l=0, \ldots, L\right\}$ satisfying $[a, b]=\bigcup_{(l, k) \in \mathcal{G}} V_{l, k}$ with $\left|V_{l, k} \cap V_{l^{\prime}, k^{\prime}}\right|=0$ for $(l, k) \neq\left(l^{\prime}, k^{\prime}\right)$. How to determine $\mathcal{G}$ is detailed in the sequel. First of all, we assume that $\mathcal{D}$ is a tree, i.e., the relation

$$
(l, k, i) \in \mathcal{D} \Rightarrow(l, k, j) \in \mathcal{D} \text { and }(l-1,\lfloor k / 2\rfloor, j) \in \mathcal{D} \quad \forall j \in \mathcal{P},
$$

holds for any $l \in\{1, \ldots, L-1\}$. Note that in the case of adaptive MR-FV schemes 35] the tree needs to be graded of degree $q \geq 1$. This leads to larger trees and, hence, degrades the efficiency. Then $\mathcal{G}$ can be determined recursively. For this purpose, the index set $\mathcal{G}$ is initialized by all indices of the coarsest discretization. Then, traversing through the levels from coarse to fine we proceed as follows: if $(l, k, i) \in \mathcal{D}$ for some $i \in \mathcal{P}$, then the cell $V_{l, k}$ is locally refined, i.e., the index $(l, k)$ is removed from $\mathcal{G}$ and the indices of the subcells on the finer level are added to $\mathcal{G}$. Finally, we obtain a locally adapted grid which naturally corresponds to the outer leaves of the tree of significant details, i.e.,

$$
\mathcal{G}=\{(l, k):(l, k, i) \notin \mathcal{D} \text { for all } i \in \mathcal{P} \text { and }(l-1,\lfloor k / 2\rfloor, i) \in \mathcal{D} \text { for some } i \in \mathcal{P}\} .
$$

This procedure is described in Algorithm 2 below together with the computation of the corresponding single-scale coefficients. See Figure 1 for an illustration of the multi-scale transformation (a), (b) and the adaptive grid (d) corresponding to the set $\mathcal{D}$, computed by the thresholding operation (c).

\section{Adaptive multiresolution DG scheme}

We now combine the MRA in Section 3 with the DG scheme presented in Section 2. The idea is first to derive evolution equations for the single-scale and 
the multi-scale coefficients, respectively, and then to apply thresholding to discard non-significant contributions and, hence, to reduce the complexity of the evolution system. The procedure is similar to the derivation of adaptive MR-FV schemes; cf. [35], p. $73 \mathrm{ff}$. The starting point is a given DG discretization that is defined on the uniform highest resolution level $L$, i.e., $v_{L}=u_{h}$ with $v_{L, k, i}=v_{k, i}$ in (2.3). This will be referred to as the reference scheme on the reference mesh. Since for the time discretization a TVD-Runge-Kutta method is used, where each stage can be interpreted as a linear combination of forward Euler steps, we confine ourselves to the forward Euler step (2.6) with (2.7), (2.8) and (2.9).

4.1. Evolution equations for single-scale and multi-scale coefficients. In a first step we now apply the two-scale relation (3.14) for the single-scale coefficients to the evolution equation (2.6), where we replace $u_{l+1,2 k+s, j}$ by $v_{l+1,2 k+s, j}^{\mu}$ for $\mu=$ $n$ and $\mu=n+1$. Proceeding levelwise from fine to coarse we then recursively obtain for any $k \in \mathcal{I}_{l}, i \in \mathcal{P}$, the two-scale evolution equations for the single-scale coefficients

$$
v_{l, k, i}^{n+1}=v_{l, k, i}^{n}-\tau\left(B_{l, k, i}^{n}-G_{l, k, i}^{n}-S_{l, k, i}^{n}\right) .
$$

It turns out that the numerical flux $F_{l, k}^{n}$, the numerical flux balance $B_{l, k, i}^{n}$, the flux integral $G_{l, k, i}^{n}$ and the source integral $S_{l, k, i}^{n}$ are recursively determined by

$$
\begin{aligned}
& F_{l, k}^{n}:=F_{l+1,2 k}^{n}=\ldots=F_{L, 2^{L-l} k}^{n}=F\left(v_{L}\left(t^{n}, x_{L, 2^{L-l} k}^{-}\right), v_{L}\left(t^{n}, x_{L, 2^{L-l} k}^{+}\right)\right), \\
& B_{l, k, i}^{n}:=F_{l, k+1}^{n} \tilde{\varphi}_{l, k, i}\left(x_{l, k+1}^{-}\right)-F_{l, k}^{n} \tilde{\varphi}_{l, k, i}\left(x_{l, k}^{+}\right), \\
& G_{l, k, i}^{n}:=\sum_{s \in\{0,1\}} \sum_{j \in \mathcal{P}} \tilde{m}_{i j}^{0, s} G_{l+1,2 k+s, j}^{n}=\int_{V_{l, k}} f\left(v_{L}\left(t^{n}, x\right)\right) \tilde{\varphi}_{l, k, i}^{\prime}(x) d x, \\
& S_{l, k, i}^{n}:=\sum_{s \in\{0,1\}} \sum_{j \in \mathcal{P}} \tilde{m}_{i j}^{0, s} S_{l+1,2 k+s, j}^{n}=\int_{V_{l, k}} s\left(v_{L}\left(t^{n}, x\right)\right) \tilde{\varphi}_{l, k, i}(x) d x
\end{aligned}
$$

The derivation is similar to the procedure in the finite volume context; cf. [35]. The details can be found in 42 , p. $59 \mathrm{ff}$.

Note that due to the nestedness of the grid hierarchy and the consistency property of the numerical fluxes, the flux balances are only computed by the fine-scale fluxes corresponding to the edges of the coarse cell; see (4.3). These, in particular, have to be determined by the fine scale data. However, the internal fluxes cancel and, hence, the overall complexity is reduced, because we only have to compute two fluxes for $V_{l, k}$ instead of $2^{L-l}+1$ fluxes corresponding to the cells $V_{L, 2^{L-l} k+r}$, $r=0, \ldots, 2^{L-l}-1$. In higher dimensions, the subdivision of the cell faces has to be taken into account. For instance, for a $d$-dimensional Cartesian grid hierarchy we would have to compute $2 d 2^{(L-l)(d-1)}$ fluxes that correspond to all interfaces on level $L$ that form a partition of an interface on a coarser scale $l$. On the other hand, the volume integrals of the flux and source integral, (4.4) and (4.5), are determined by the sum of all integrals on the finest scale $L$ according to (2.9). Hence there is no complexity reduction, i.e., we still have the complexity of the reference grid. However, if the integrals can be computed exactly, then this sum can be replaced by one integral on the local scale $l$.

In a second step, we similarly derive evolution equations for the details where we recursively apply the two-scale transformation (3.14) for the detail coefficients to the evolution equations (4.1) of the single-scale coefficients for $l=L-1, \ldots, 0$. 
Then the evolution process (2.6) on the uniform reference mesh is equivalent to the evolution of the multi-scale coefficients, i.e., coarse-scale coefficients and details,

$$
\begin{aligned}
& v_{0, k, i}^{n+1}=v_{0, k, i}^{n}-\tau\left(B_{0, k, i}^{n}-G_{0, k, i}^{n}-S_{0, k, i}^{n}\right), \\
& d_{l, k, i}^{n+1}=d_{l, k, i}^{n}-\tau\left(\bar{B}_{l, k, i}^{n}-\bar{G}_{l, k, i}^{n}-\bar{S}_{l, k, i}^{n}\right) .
\end{aligned}
$$

Here the numerical flux balance, the flux integral and the source integral differ from those in (4.1):

$$
\begin{aligned}
& \bar{B}_{l, k, i}^{n}:=\sum_{j \in \mathcal{P}} \sum_{s \in\{0,1\}} \tilde{m}_{i, j}^{1, s} B_{l+1,2 k+s, j}^{n} \\
& =F_{l, k+1}^{n} \tilde{\psi}_{l, k, i}\left(x_{l, k+1}^{-}\right)-F_{l, k}^{n} \tilde{\psi}_{l, k, i}\left(x_{l, k}^{+}\right)-\left.F_{l+1,2 k+1}^{n} \tilde{\psi}_{l, k, i}(x)\right|_{x_{l+1,2 k+1}^{-}} ^{x_{l+2 k+1}^{+}}, \\
& \text {(4.9) } \bar{G}_{l, k, i}^{n}:=\sum_{j \in \mathcal{P}} \sum_{s \in\{0,1\}} \tilde{m}_{i, j}^{1, s} G_{l+1,2 k+s, j}^{n}=\sum_{s \in\{0,1\}} \int_{V_{l+1,2 k+s}} f\left(v_{L}\left(t^{n}, x\right)\right) \tilde{\psi}_{l, k, i}^{\prime}(x) d x \text {, } \\
& \text { (4.10) } \bar{S}_{l, k, i}^{n}:=\sum_{j \in \mathcal{P}} \sum_{s \in\{0,1\}} \tilde{m}_{i, j}^{1, s} S_{l+1,2 k+s, j}^{n}=\int_{V_{l, k}} s\left(v_{L}\left(t^{n}, x\right)\right) \tilde{\psi}_{l, k, i}(x) d x \text {. }
\end{aligned}
$$

In particular, we note that the multiwavelet $\psi_{l, k, i}$ typically jumps at the cell center $x_{l, k+\frac{1}{2}}=x_{l+1,2 k+1}$. Therefore the internal numerical flux $F_{l+1,2 k+1}^{n}$ does not cancel in (4.8) and we have to split the integral in (4.9) into two parts. Again, the derivation is similar to the procedure in the finite volume context; cf. 35. The main difference is caused by the discontinuity of the multiwavelets inside a cell. For details we refer to 42 , p. $59 \mathrm{ff}$.

According to the change of basis (3.12) applied to (2.3), the evolution step (2.6) of the reference scheme can be rewritten as

$$
v_{L}^{n+1}=\sum_{k \in \mathcal{I}_{0}} \sum_{i \in \mathcal{P}} v_{0, k, i}^{n+1} \varphi_{0, k, i}+\sum_{l=0}^{L-1} \sum_{k \in \mathcal{I}_{l}} \sum_{i \in \mathcal{P}} d_{l, k, i}^{n+1} \psi_{l, k, i},
$$

where the multi-scale coefficients are determined by (4.6) and (4.7).

As is motivated by the cancellation property (3.17), we may reduce the degrees of freedom by first applying data compression to the multi-scale coefficients without significant loss of accuracy. Then the compressed set of equations is solved on a locally refined adaptive grid characterized by some index set $\mathcal{G}$. According to Section 3 this set is determined by means of an index set $\mathcal{D}$ corresponding to significant detail coefficients. Note that, if $\mathcal{D}$ is a tree, the system of evolution equations for the single-scale coefficients (4.1) for $\mathcal{G}$ is equivalent to the system of evolution equations for the multi-scale coefficients (4.6) and (4.7), i.e., by the sets $\mathcal{D}$ and $\mathcal{G}$ a local change of basis is performed that, in analogy to (3.12), reads

$$
v_{L}^{n+1}=\sum_{(l, k) \in \mathcal{G}} \sum_{i \in \mathcal{P}} v_{l, k, i}^{n+1} \varphi_{l, k, i}=\sum_{k \in \mathcal{I}_{0}} \sum_{i \in \mathcal{P}} v_{0, k, i}^{n+1} \varphi_{0, k, i}+\sum_{(l, k, i) \in \mathcal{D}} d_{l, k, i}^{n+1} \psi_{l, k, i} .
$$

The switch between the two representations characterized by the local single-scale coefficients $\mathbf{v}_{\mathcal{G}}=\left(v_{l, k, i}\right)_{(l, k) \in \mathcal{G}, i \in \mathcal{P}}$ and the compressed multi-scale coefficients $\mathbf{v}_{\mathcal{D}}=$ $\left(\left(v_{0, k, i}\right)_{k \in \mathcal{I}_{0}, i \in \mathcal{P}},\left(d_{l, k, i}\right)_{(l, k, i) \in \mathcal{D}}\right)$, respectively, can be realized by applying Algorithms 1 and 2 ,

Algorithm 1 (Local transformation from single-scale to multi-scale coefficients). Let $\mathcal{G}$ characterize an adaptive grid with associated local single-scale coefficients 
$\mathbf{v}_{\mathcal{G}}=\left(v_{l, k, i}\right)_{(l, k) \in \mathcal{G}, i \in \mathcal{P}}$. Then the corresponding tree $\mathcal{D}$ and its compressed multiscale coefficients $\mathbf{d}_{\mathcal{D}}=\left(\left(v_{0, k, i}\right)_{k \in \mathcal{I}_{0}, i \in \mathcal{P}},\left(d_{l, k, i}\right)_{(l, k, i) \in \mathcal{D}}\right)$ can be computed as follows: Step 1. Initialize the set $\mathcal{D}=\{(L-1,\lfloor k / 2\rfloor, i):(L, k) \in \mathcal{G}, i \in \mathcal{P}\}$.

Step 2. For $l=L-1$ down to 0 do for $(l, k, i) \in \mathcal{D}$ do

(1) compute $v_{l, k, i}$ and $d_{l, k, i}$ according to (3.14);

(2) if $l \neq 0$ then add $(l-1,\lfloor k / 2\rfloor, i)$ to $\mathcal{D}$ for all $i \in \mathcal{P}$.

Algorithm 2 (Local transformation from multi-scale to single-scale coefficients). Let $\mathcal{D}$ be a tree and $\mathbf{d}_{\mathcal{D}}=\left(\left(v_{0, k, i}\right)_{k \in \mathcal{I}_{0}, i \in \mathcal{P}},\left(d_{l, k, i}\right)_{(l, k, i) \in \mathcal{D}}\right)$ the array containing the compressed multi-scale coefficients. Then the adaptive grid corresponding to the set $\mathcal{G}$ and its local single-scale coefficients $\mathbf{v}_{\mathcal{G}}=\left(v_{l, k, i}\right)_{(l, k) \in \mathcal{G}, i \in \mathcal{P}}$ can be computed as follows:

Step 1. Initialize the set $\mathcal{G}=\left\{(0, k): k \in \mathcal{I}_{0}\right\}$ and the array $\mathbf{v}_{\mathcal{G}}=\left(v_{0, k, i}\right)_{k \in \mathcal{I}_{0}, i \in \mathcal{P}}$. Step 2. For $l=0$ to $L-1$ do If $(l, k, j) \in \mathcal{D}$ for some $j \in \mathcal{P}$, then:

(1) compute $v_{l+1,2 k+s, i}, i \in \mathcal{P}, s \in\{0,1\}$, according to 3.15 and add them to the array $\mathbf{v}_{\mathcal{G}}$;

(2) add the indices $(l+1,2 k+s), s \in\{0,1\}$, to the set $\mathcal{G}$;

(3) remove the index $(l, k)$ from the set $\mathcal{G}$;

(4) discard the coefficients $v_{l, k, i}, i \in \mathcal{P}$, in the array $\mathbf{v}_{\mathcal{G}}$.

Note that by applying Algorithm 1 without Step 2.1 and Algorithm 2 without Step 2.1 and 2.4 can be used to compute the index set $\mathcal{D}$ from the index set $\mathcal{G}$ and vice versa.

From a practical point of view, it is preferable to evolve the cell averages according to (4.1) on a locally refined grid, because the local single-scale coefficients are needed in the limiting process. Moreover, we need to evaluate $v_{L}$ when computing the numerical flux (4.2) as well as the flux integral (4.4) and source integral (4.5). For this purpose, the evaluation of the single-scale representation is more efficient.

So far, we have specified the equations to be solved by the adaptive MR-DG scheme. It remains to explain (i) how to determine $\mathcal{D}$ and $\mathcal{G}$, respectively, from the data of the previous time step and (ii) how to perform limiting on the adaptive grid.

4.2. Prediction. The idea of the adaptive multiresolution DG scheme is to perform the evolution step only for significant details

$$
\mathcal{D}^{n+1}:=\left\{(l, k, i):\left|d_{l, k, i}^{n+1}\right|>\varepsilon_{l}, i \in \mathcal{P}, k \in \mathcal{I}_{l}, l \in\{0, \ldots, L-1\}\right\}
$$

and to discard all other equations. Since this set cannot be computed before the data at time level $t^{n+1}$ are known, a prediction set $\tilde{\mathcal{D}}^{n+1}$ has to be computed from $\mathcal{D}^{n}$ such that the reliability condition

$$
\mathcal{D}^{n} \cup \mathcal{D}^{n+1} \subset \tilde{\mathcal{D}}^{n+1}
$$

holds.

Obviously, the efficiency of the adaptive MR-DG scheme crucially relies on the compression rate of the threshold process. However, if we miss a significant detail in the prediction step, then the threshold error might dominate the discretization error of the reference DG scheme. Therefore the prediction set has to be designed such 
that all detail coefficients not contained in the prediction set remain non-significant after the update including the evolution step and the limiting process.

In the context of the adaptive MR-FV scheme, a prediction strategy has been developed for which the reliability condition (4.13) could be analytically verified; see [18, 28. This strategy cannot be applied to the adaptive MR-DG scheme because of the limiting process and missing regularity of the primal multiwavelet functions. We therefore had to design a new prediction strategy summarized in Algorithm 3 . A proof of the reliability condition (4.13) is given later on in Section 6.3, where the Steps E1-E3 and the Steps L4-L6 will be employed to verify reliability corresponding to time evolution (E) and limiting (L), respectively. In particular, these steps imply some properties to be proven in Lemma 1 that are employed later on in Lemma 2, Corollary 2 and 3 as well as Theorem 3 .

Algorithm 3 (Construction of prediction set). Let

$$
\mathbf{d}_{\mathcal{D}}=\left(\left(v_{0, k, i}\right)_{k \in \mathcal{I}_{0}, i \in \mathcal{P}},\left(d_{l, k, i}\right)_{(l, k, i) \in \mathcal{D}}\right)
$$

be the multi-scale coefficients corresponding to some set $\mathcal{D}$ and $v_{L}$ the function spanned by these coefficients according to (4.12). To compute the prediction set $\tilde{\mathcal{D}}=\tilde{\mathcal{D}}\left(\mathbf{d}_{\mathcal{D}}\right)$ we perform the following nine steps:

Step 0 (Initial inclusion of $\mathcal{D}_{0}$ ). The prediction set has to include all indices corresponding to non-vanishing detail coefficients of the data $\mathbf{d}_{\mathcal{D}}$ contained in the support $\mathcal{D}_{0}=\left\{(l, k, i):\left|d_{l, k, i}\right|>0\right\}$, i.e., $\tilde{\mathcal{D}} \leftarrow \mathcal{D}_{0}$.

Then, the Steps E1-E3 and $L 4-L 6$ are performed for any index $(l, k) \in \mathcal{G}_{0}$ corresponding to all cells of the current grid:

Step E1 (Jump at cell interfaces). The jump at the left and right cell interface is determined, i.e., $J_{L}:=\left|v_{L}\left(x_{l, k}^{+}\right)-v_{L}\left(x_{l, k}^{-}\right)\right|$and $J_{R}:=\left|v_{L}\left(x_{l, k+1}^{+}\right)-v_{L}\left(x_{l, k+1}^{-}\right)\right|$. If the jump at $x_{l, k}$ is larger than $\varepsilon$, we add the left and right cell neighbors attached to the grid point $x_{l, k}$ on level $L$ to the adaptive grid. This is enforced by the inclusion of the corresponding details on level $L-1$ to the prediction set, i.e.,

$$
\tilde{\mathcal{D}} \leftarrow \tilde{\mathcal{D}} \cup\left\{\left(L, 2^{L-l-1} k-1, i\right): i \in \mathcal{P}\right\} \cup\left\{\left(L, 2^{L-l-1} k, i\right): i \in \mathcal{P}\right\} .
$$

Similarly we proceed with $J_{R}$ and $x_{l, k+1}$.

Step E2 (Flux integral bound). For each cell $(l, k) \in \mathcal{G}_{0}$ we calculate bounds $D_{v}^{r}$ for the rth derivative of $v_{L}$ in $V_{l, k}$, i.e.,

$$
\left|\frac{d^{r}}{d x^{r}} v_{L}(x)\right| \leq h_{l}^{-r} \sum_{i \in \mathcal{P}}\left|v_{l, k, i}\right| D_{r, i}=: D_{v}^{r}, \quad \forall x \in V_{l, k},
$$

where the constants $D_{r, i}$ are determined according to (3.16). Then we may bound the $(p+1)$ st derivative of the flux $f$ via chain rule by

$$
\left\|\frac{d^{p+1}}{d x^{p+1}} f\left(v_{L}(t, \cdot)\right)\right\|_{L^{\infty}\left(V_{l, k}\right)} \leq \sum_{\mathbf{k} \in T_{p+1}} \frac{(p+1) !}{k_{1} ! \cdots k_{p+1} !} D_{f}^{|\mathbf{k}|} \prod_{j=1, k_{j} \geq 1}^{p+1}\left(\frac{D_{v}^{j}}{j !}\right)^{k_{j}},
$$

where the set $T_{p+1}$ contains all $(p+1)$-tupels $\mathbf{k}=\left(k_{1}, \ldots, k_{p+1}\right)$ of non-negative integers such that $\sum_{i=1}^{p+1} i k_{i}=p+1$ and $|\mathbf{k}|=\sum_{i=1}^{p+1} k_{i}$. The constant $D_{f}^{r}$ is a problem-dependent bound for the rth derivative of the flux, i.e.,

$$
D_{f}^{r}:=\max _{|u| \leq C_{\infty}}\left|D^{r} f(u)\right|,
$$


where $C_{\infty}=C_{\infty}\left(T, u_{0}, s\right)$ is determined in Proposition 3. Then we determine a level

$$
l^{\prime} \geq \log _{2^{p+1}}\left(\frac{1}{\varepsilon}\left(h_{0} / 2\right)^{p} \sum_{\mathbf{k} \in T_{p+1}} \frac{p+1}{k_{1} ! \cdots k_{p+1} !} D_{f}^{|\mathbf{k}|} \prod_{j=1, k_{j} \geq 1}^{p+1}\left(\frac{D_{v}^{j}}{j !}\right)^{k_{j}}\right)
$$

depending on the polynomial degree $p$ of the basis functions and include all subcells $V_{l^{\prime}, k^{\prime}} \subset V_{l, k}$ of $V_{l, k}$ on higher refinement levels, i.e.,

$$
\tilde{\mathcal{D}} \leftarrow \tilde{\mathcal{D}} \cup\left\{\left(l^{\prime}, k^{\prime}, i\right): V_{l^{\prime}, k^{\prime}} \subset V_{l, k}, i \in \mathcal{P}\right\} .
$$

Step E3 (Source integral bound). We proceed similarly for derivatives of the source function $s$, but only up to the order $p$, i.e.,

$$
l^{\prime} \geq \log _{2^{p+1}}\left(\frac{1}{\varepsilon}\left(h_{0} / 2\right)^{p} \sum_{\mathbf{k} \in T_{p}} \frac{1}{k_{1} ! \cdots k_{p} !} D_{s}^{|\mathbf{k}|} \prod_{j=1, k_{j} \geq 1}^{p}\left(\frac{D_{v}^{j}}{j !}\right)^{k_{j}}\right)
$$

with $D_{s}^{|\mathbf{k}|}$ instead of $D_{f}^{|\mathbf{k}|}$.

Step L4 (Limiting at cell interfaces). Let $k^{+}=2^{(L-l)} k$ and $k^{-}=2^{(L-l)}(k+$ $1)-1$. We calculate the mean values $v_{L, k^{+}-1,0}, v_{L, k^{+}, 0}$ and $v_{L, k^{-}, 0}, v_{L, k^{-}+1,0}$ of the neighbors on level $L$ to the cell interfaces at $x_{l, k}$ and $x_{l, k+1}$, respectively. An efficient procedure for this is described in [4], Section 4.6.2. Then we evaluate $v_{L}$ at the cell interfaces and compute $c_{L, k^{ \pm}}^{ \pm}$.

First of all we consider the left cell interface $x_{l, k}^{+}$: If $c_{L, k^{+}}^{ \pm} \neq 1$, the limiter changes the data and we check whether

$$
\left|v_{L}\left(x_{l, k}^{+}\right)-v_{L, k^{+}, 0}\right| \geq \varepsilon \text { or }\left|v_{L}\left(x_{L, k^{+}+1}\right)-v_{L, k^{+}, 0}\right| \geq \varepsilon .
$$

If this holds true, then limiting the data on the cell $V_{L, k^{+}}$might introduce an error larger than $\varepsilon$. Therefore, we enforce refinement up to level $L$, i.e.,

$$
\tilde{\mathcal{D}} \leftarrow \tilde{\mathcal{D}} \cup\left\{\left(L, k^{+}, i\right): i \in \mathcal{P}\right\} .
$$

The same check is performed at the right cell interface $x_{l, k+1}^{-}$: if the limiter modifies the data, i.e., $c_{L, k^{-}}^{ \pm} \neq 1$ and

$$
\left|v_{L}\left(x_{l, k+1}^{-}\right)-v_{L, k^{-}, 0}\right| \geq \varepsilon \text { or }\left|v_{L}\left(x_{L, k^{-}}\right)-v_{L, k^{-}, 0}\right| \geq \varepsilon,
$$

then we refine up to level $L$, i.e.,

$$
\tilde{\mathcal{D}} \leftarrow \tilde{\mathcal{D}} \cup\left\{\left(L, k^{-}, i\right): i \in \mathcal{P}\right\} .
$$

Step L5 (Limiting inside of cells). We first find an upper bound for the second derivative of $v_{L}$ (see Step E2)

$$
\left|v_{L}^{\prime \prime}(x)\right| \leq D_{v}^{2}, \quad x \in V_{l, k} .
$$

Then we must have the bound $D_{v}^{2} \leq C_{L} \varepsilon h_{L}^{-2}$, otherwise we refine up to level $L$, i.e.,

$$
\tilde{\mathcal{D}} \leftarrow \tilde{\mathcal{D}} \cup\left\{\left(L, k^{\prime}, i\right): V_{L, k^{\prime}} \subset V_{l, k}, i \in \mathcal{P}\right\} .
$$

Step L6 (Entropy bound on the gradient). We find an upper bound for the first derivative of $v_{L}$ (see Step E2)

$$
\left|v_{L}^{\prime}(x)\right| \leq D_{v}^{1}, \quad x \in V_{l, k} .
$$


Then we check if $D_{v}^{1} \leq C_{\alpha} h_{L}^{\alpha-1}$, where $C_{\alpha}$ and $\alpha$ stem from the entropy correction of the limiter; see (2.10b). If the estimate does not hold, we refine up to level $L$, i.e.,

$$
\tilde{\mathcal{D}} \leftarrow \tilde{\mathcal{D}} \cup\left\{\left(L, k^{\prime}, i\right): V_{L, k^{\prime}} \subset V_{l, k}, i \in \mathcal{P}\right\}
$$

Step B7 (Additional refinement of neighbors to level $L$ ). The set $\tilde{\mathcal{D}}$ determined by the previous steps characterizes an adaptive grid $\tilde{\mathcal{G}}$ applying Algorithm 2 . The cells in $\tilde{\mathcal{G}}$ on the highest refinement level are given by

$$
\Sigma(\tilde{\mathcal{D}}, L):=\{(L, k):(L-1,\lfloor k / 2\rfloor, i) \in \tilde{\mathcal{D}} \text { for some } i \in \mathcal{P}\}=\{(L, k) \in \tilde{\mathcal{G}}\} .
$$

Then we enlarge the prediction set $\tilde{\mathcal{D}}$ such that all neighbors of cells on level $L$ characterized by

$$
\Sigma^{\partial}:=\{(L, k) \notin \Sigma(\tilde{\mathcal{D}}, L):(L, k-1) \in \Sigma(\tilde{\mathcal{D}}, L) \text { or }(L, k+1) \in \Sigma(\tilde{\mathcal{D}}, L)\}
$$

are included in the resulting adaptive grid, i.e.,

$$
\tilde{\mathcal{D}} \leftarrow \tilde{\mathcal{D}} \cup\left\{(L-1,\lfloor k / 2\rfloor, i):(L, k) \in \Sigma^{\partial}, i \in \mathcal{P}\right\} .
$$

Step 8 (Ensure tree property). Enlarge the set $\tilde{\mathcal{D}}$, such that the tree property (3.23) holds.

Remark 1. Since by Step E1 grid refinement is triggered up to the highest level, this seems to be demanding. However, if the solution is locally smooth, then this jump converges to zero when the discretization becomes finer, i.e., $L$ is increasing. In all of the numerous computations reported in 42, 29] for scalar problems (convex, nonconvex flux function) and the Euler system, this condition did not trigger too much refinement except for threshold values much smaller than the optimal threshold value. Moreover, the tree corresponding to $\mathcal{D}$ must not be graded. Therefore grid refinement is only triggered by Step E1 in the two neighboring cells of a cell interface.

Remark 2. In Section 6 we need to determine for data $\mathbf{w} \in\left(\mathbb{R}^{p}\right)^{N_{L}}$ the prediction set $\tilde{\mathcal{D}}(\mathbf{w})$ and the corresponding adaptive grid characterized by the set $\tilde{\mathcal{G}}(\mathbf{w})$. These sets can be determined by (i) performing a multiresolution analysis, i.e., applying Algorithm 1 with $\mathbf{v}_{\mathcal{G}}=\mathbf{w}$ and $\mathcal{G}=\{L\} \times \mathcal{I}_{L}$ to compute $\mathbf{d}_{\mathcal{D}}$ and $\mathcal{D}$, (ii) computing the prediction set $\tilde{\mathcal{D}}=\tilde{\mathcal{D}}(\mathbf{w})$ by applying Algorithm 3 with $\mathbf{d}_{\mathcal{D}}$ and $\mathcal{D}$ and (iii) determining the adaptive grid $\tilde{\mathcal{G}}(\mathbf{w})$ and corresponding data $\mathbf{w}_{\tilde{\mathcal{G}}}(\mathbf{w})$ by applying Algorithm 2 with $\mathcal{D}$ and $\mathbf{d}_{\tilde{\mathcal{D}}}$, where $d_{l, k, i}=0$ for $(l, k, i) \in \tilde{\mathcal{D}} \backslash \mathcal{D}$. In particular, this procedure becomes effective when applied to thresholded data, i.e., $\mathbf{w}=\mathcal{A}_{\varepsilon} \mathbf{v}$, $\mathbf{v} \in\left(\mathbb{R}^{p}\right)^{N_{L}}$.

4.3. Limiting. In order to avoid oscillations near to discontinuities we apply a limiter to the data of the adaptive grid. According to Section 3 the adaptive grid is determined by the prediction set $\tilde{\mathcal{D}}^{n+1}$. Since by the multi-scale analysis discontinuities will be characterized by significant detail coefficients, the grid is locally refined up to the finest scale $L$. In all other regions the solution is locally smooth and the corresponding details might be negligibly small. Therefore we may expect that the grid is locally coarse. Hence, limiting has only to be performed on the data of the highest refinement level. 
Algorithm 4 (Adaptive limiter). Let $\mathbf{v}_{\tilde{\mathcal{G}}}=\left(v_{l, k, i}\right)_{(l, k) \in \tilde{\mathcal{G}}, i \in \mathcal{P}}$ be the local singlescale coefficients on the adaptive grid $\tilde{\mathcal{G}}$ determined by some prediction set $\tilde{\mathcal{D}}$. Then the limited values $\bar{v}_{l, k, i}, i \in \mathcal{P}$, are locally determined for $(l, k) \in \tilde{\mathcal{G}}$ by the following procedure:

Case 1 (Highest level: $l=L$ ). If the cell $V_{L, k}$ has a neighboring cell that is not on the same level $L$, it stems from Step B7 in the prediction strategy. In that case, we do nothing. Otherwise we apply the reference limiter (2.10).

Case 2 (Lower level: $l<L$ ). No limiting is performed at all, i.e., $\bar{v}_{l, k, i}=v_{l, k, i}$, $i \in \mathcal{P}$.

Remark 3. Note that because of Step B7 in Algorithm 3 the limiting process gives the same result in Case 1 as the limiter applied to the data corresponding to the reference mesh. In particular, we avoid the fact that the limiter involves data on different levels. This fact is needed in Corollary 3.

4.4. Adaptive MR-DG scheme. Finally, we summarize the adaptive MR-DG scheme in the following algorithm. It basically consists of three steps corresponding to (i) refinement, (ii) limiting and evolution, as well as (iii) coarsening. For $n=0$ the algorithm can be initialized by (i) projecting the initial data $u_{0}$ to the reference mesh, (ii) performing the multi-scale transformation to the coefficients, (iii) applying hard thresholding and (iv) performing a local inverse multi-scale transformation. However, to avoid the complexity of the reference mesh, one should apply the bottom-up strategy proposed in [35, where we successively proceed from coarse to fine scale.

Algorithm 5 (Adaptive MR-DG scheme). Let $\mathcal{G}^{n}$ characterize the adaptive grid at time $t_{n}$ with associated local single-scale coefficients $\mathbf{v}_{\mathcal{G}^{n}}=\left(v_{l, k, i}^{n}\right)_{(l, k) \in \mathcal{G}^{n}, i \in \mathcal{P}}$. Then we perform the following three steps to update the data and the grid at time $t_{n+1}$ :

Step 1 (Refinement).

(a) We apply Algorithm 1 with $\mathcal{G}=\mathcal{G}^{n}$ and $\mathbf{v}_{\mathcal{G}}=\mathbf{v}_{\mathcal{G}^{n}}$ to compute the compressed multi-scale coefficients $\mathbf{d}_{\mathcal{D}^{n}}$ and $\mathcal{D}^{n}$.

(b) We apply Algorithm 3 with $\mathbf{d}=\mathbf{d}_{\mathcal{D}^{n}}$ and $\mathcal{D}=\mathcal{D}^{n}$ to determine the prediction set $\tilde{\mathcal{D}}^{n+1}$.

(c) We apply Algorithm 2 with $\tilde{\mathcal{D}}^{n+1}$ and $\mathbf{d}_{\tilde{\mathcal{D}}^{n+1}}$, where we put the detail coefficients corresponding to $\tilde{\mathcal{D}}^{n+1} \backslash \mathcal{D}^{n}$ to zero, i.e., we determine the predicted grid $\tilde{\mathcal{G}}^{n+1}$ and the associated local single-scale coefficients $\mathbf{v}_{\tilde{\mathcal{G}}^{n+1}}=$ $\left(v_{l, k, i}^{n}\right)_{(l, k) \in \tilde{\mathcal{G}}^{n+1}, i \in \mathcal{P}}$.

Step 2a (Limiting). We apply the limiter from Algorithm 4 to the data $\mathbf{v}_{\tilde{\mathcal{G}}^{n+1}}$ on the adaptive grid $\tilde{\mathcal{G}}^{n+1}$, where only higher order coefficients on the highest resolution level are modified; the limited data are $\overline{\mathbf{v}}_{\tilde{\mathcal{G}}^{n+1}}$.

Step 2b (Evolution). We perform time evolution of the local single-scale coefficients corresponding to the adaptive grid $\tilde{\mathcal{G}}^{n+1}$ applying the evolution equations (4.1) for $(l, k) \in \tilde{\mathcal{G}}^{n+1}, i \in \mathcal{P}$, i.e., we determine $\tilde{\mathbf{v}}_{\tilde{\mathcal{G}}^{n+1}}$.

Step 3 (Coarsening).

(a) We apply Algorithm 1 with $\mathcal{G}=\tilde{\mathcal{G}}^{n+1}$ and $\tilde{\mathbf{v}}_{\tilde{\mathcal{G}}^{n+1}}$ to compute the compressed multi-scale coefficients $\tilde{\mathbf{d}}_{\tilde{\mathcal{D}}^{n+1}}$ and $\tilde{\mathcal{D}}^{n+1}$. 
(b) We apply hard thresholding to $\tilde{\mathbf{d}}_{\tilde{\mathcal{D}}^{n+1}}$ and $\tilde{\mathcal{D}}^{n+1}$, where we discard all detail coefficients with $\left|d_{l, k, i}\right| \leq 0$ for all $i \in \mathcal{P}$ to determine $\mathcal{D}^{n+1}$ and $\mathbf{d}_{\mathcal{D}^{n+1}}$.

(c) We apply Algorithm 2 with $\mathcal{D}^{n+1}$ and $\mathbf{d}_{\mathcal{D}^{n+1}}$, where we put the detail coefficients corresponding to $\tilde{\mathcal{D}}^{n+1} \backslash \mathcal{D}^{n+1}$ to zero, i.e., we determine the new adaptive grid $\mathcal{G}^{n+1}$ and the associated local single-scale coefficients $\mathbf{v}_{\mathcal{G}^{n+1}}=\left(v_{l, k, i}^{n+1}\right)_{(l, k) \in \mathcal{G}^{n+1}, i \in \mathcal{P}}$.

For an efficient implementation one may employ hash maps (cf. [37, 7]), similar to those in the context of adaptive MR-FV schemes [35].

\section{ERror ANALYSIS: FrAMEWORK FOR ADAPTIVE SCHEMES}

The objective of the proposed adaptive MR-DG scheme is to reduce computational cost and memory requirements for a given DG scheme performed on the uniform reference mesh while preserving the accuracy of the reference scheme. Since grid adaptation is triggered only by the threshold value $\varepsilon$, we need to propose a strategy on how to choose $\varepsilon$. For this purpose we use a perturbation argument motivated by the perturbation analysis for adaptive MR-FV schemes, cf. [35], where we interpret the adaptive scheme as a perturbation of the reference scheme. First of all, here we describe a general framework on how to control a perturbation introduced into a given DG scheme. This will provide us with sufficient conditions to estimate the perturbation error for the adaptive MR-DG scheme presented in Section 4. These are verified in Section 6.

We start by introducing the entropy solution $u$ of the initial value problem (2.1) and $u_{h} \in S^{p}$ defined according to (2.3) with $v_{k, i}(t)=\left\langle u(t, \cdot), \tilde{\varphi}_{k, i}\right\rangle_{\Omega}$. Let $v$ be the approximate solution function determined by the reference DG scheme presented in Section 2. By $v_{\varepsilon}$ we denote the approximate solution function of some other scheme, for instance, an adaptive scheme where the local data are prolongated to the reference mesh identified by the index set $\mathcal{I}$ with discretization $h=(b-a) / N$. This is referred to as an adaptive scheme in the sequel, but it is not necessarily determined by the adaptive MR-DG scheme in Section 4, where $h=(b-a) / N_{L}=$ $h_{L}$ and $\mathcal{I}=\mathcal{I}_{L}$. Here $\varepsilon$ denotes a parameter that controls the perturbation such that for $\varepsilon \rightarrow 0$ the adaptive scheme coincides with the reference scheme. In the context of adaptive MR-DG schemes this parameter can be identified with the threshold value. An ideal strategy would be to prescribe an error tolerance TOL and then choose the mesh size $h$ and the perturbation parameter $\varepsilon$ such that the error meets the tolerance, i.e.,

$$
\left\|\hat{u}(t, \cdot)-\hat{v}_{\varepsilon}(t, \cdot)\right\|_{L^{1}(\Omega)} \leq T O L .
$$

Here $\hat{w}$ denotes the piecewise mean of a function $w \in S^{p}$ according to (2.14). Since in general no error estimator is available for the adaptive scheme, we split the error into two parts corresponding to the discretization error $\hat{\eta}:=\hat{u}-\hat{v}$ of the reference DG scheme and the perturbation error $\hat{e}:=\hat{v}-\hat{v}_{\varepsilon}$ of the adaptivity, i.e.,

$$
\left\|\hat{u}(t, \cdot)-\hat{v}_{\varepsilon}(t, \cdot)\right\|_{L^{1}(\Omega)} \leq\|\hat{\eta}(t, \cdot)\|_{L^{1}(\Omega)}+\|\hat{e}(t, \cdot)\|_{L^{1}(\Omega)} \leq T O L .
$$

We now assume that there is an a priori error estimate of the discretization error for the reference scheme, i.e., $\|\hat{\eta}(t, \cdot)\|_{L^{1}(\Omega)} \sim h^{\beta}$, where $h$ denotes the spatial step size and $\beta$ the convergence order. Then, ideally we would determine $h$ such that $h^{\beta} \sim T O L$. In order to preserve the accuracy of the reference DG scheme we may 
now admit a perturbation error which is proportional to the discretization error, i.e., $\|\hat{e}(t, \cdot)\|_{L^{1}(\Omega)} \sim\|\hat{\eta}(t, \cdot)\|_{L^{1}(\Omega)}$. From this, we conclude

$$
h=h(t o l, \beta) \quad \text { and } \quad \varepsilon=\varepsilon(h) .
$$

Therefore the task is to verify an a priori estimate for the perturbation error. For this purpose we rewrite the schemes in terms of discrete operators: let us denote by $\tilde{\mathbf{v}}^{n}=\Pi \mathbf{v}^{n}$ the limiting according to (2.10) and by $\mathbf{v}^{n+1}=\mathcal{E} \tilde{\mathbf{v}}^{n}$ the evolution in time according to (2.6). Introducing the step operator $\mathcal{H}:\left(\mathbb{R}^{p}\right)^{N} \rightarrow\left(\mathbb{R}^{p}\right)^{N}$ defined by $\mathcal{H}=\mathcal{E} \Pi$ we can write one explicit Euler step of the reference scheme as $\mathbf{v}^{n+1}=\mathcal{H} \mathbf{v}^{n}$. In the following this step will be referred to as an update. In a similar way, for the adaptive scheme we introduce the step operator $\mathcal{H}_{\varepsilon}:\left(\mathbb{R}^{p}\right)^{N} \rightarrow\left(\mathbb{R}^{p}\right)^{N}$ as $\mathbf{v}_{\varepsilon}^{n+1}=\mathcal{H}_{\varepsilon} \mathbf{v}_{\varepsilon}^{n}$.

Furthermore, for any average $\hat{w}$ of a function $w \in S^{p}$ that is defined by (2.14), we define the discrete norms

$$
\begin{aligned}
& \|\hat{w}\|_{L^{q}(\Omega)}^{q}=h \sum_{k \in \mathcal{I}}\left|w_{k, 0}\right|^{q}=:\|\mathcal{R} \mathbf{w}\|_{q}^{q}, \quad q \in[1, \infty), \\
& \|\hat{w}\|_{L^{\infty}(\Omega)}=\max _{k \in \mathcal{I}}\left|w_{k, 0}\right|=:\|\mathcal{R} \mathbf{w}\|_{\infty} .
\end{aligned}
$$

Here $\mathcal{R}:\left(\mathbb{R}^{p}\right)^{N} \rightarrow \mathbb{R}^{N}$ is the restriction of a vector of higher order coefficients $\mathbf{w}=\left(w_{k, i}\right)_{k \in \mathcal{I}, i \in \mathcal{P}}$ to the vector of mean values $\mathcal{R} \mathbf{w}:=\left(w_{k, 0}\right)_{k \in \mathcal{I}}$. Note that we may estimate the $L^{q}$-norm, $q \in[1, \infty)$, by the $L^{\infty}$-norm, because the domain $\Omega=[a, b]$ is bounded. With that notation the perturbation error $\hat{e}$ is given by

$$
\left\|\hat{e}\left(t^{n}, \cdot\right)\right\|_{L^{1}(\Omega)}=\left\|\mathcal{R}\left(\mathbf{v}^{n}-\mathbf{v}_{\varepsilon}^{n}\right)\right\|_{1}=h \sum_{k \in \mathcal{I}}\left|v_{k, 0}^{n}-v_{\varepsilon, k, 0}^{n}\right| .
$$

5.1. Assumptions on adaptive and reference DG schemes. For the course of the error analysis in this section we now assume that the following properties hold for the adaptive scheme.

Assumption 1 (Properties of the adaptive scheme in the mean). (i) The error in one update step can be estimated by

$$
\left\|\mathcal{R}\left(\mathcal{H}_{\varepsilon} \mathbf{w}-\mathcal{H} \mathbf{w}\right)\right\|_{\infty} \leq C_{1}\left(h^{\gamma}+\varepsilon\right),
$$

with $\gamma>1$ for all $\mathbf{w} \in\left(\mathbb{R}^{p}\right)^{N}$ with $\|\mathcal{R} \mathbf{w}\|_{\infty} \leq C_{\infty}$.

(ii) The initial data are a perturbation of the initial data of the reference scheme

$$
\left\|\mathcal{R}\left(\mathbf{v}_{\varepsilon}^{0}-\mathbf{v}^{0}\right)\right\|_{\infty} \leq C_{2} \varepsilon .
$$

In particular, the constants $C_{1}, C_{2}$ and $C_{\infty}$ are independent of $h, \varepsilon$.

The conditions (5.6) and (5.7) will be established in Section 6 for our adaptive MR-DG scheme developed in Section 4.

Remark 4. The term $h^{\gamma}$ in (5.6) will not be present in case of the adaptive MRDG scheme in Section 6. It is needed in the analysis if the integrals in equations (4.4), (4.5) and (4.9), (4.10), respectively, are approximated by quadrature rules; see Section 6 in 42 .

Remark 5. For the adaptive MR-DG scheme described in Section 4 the constants $C_{1}, C_{2}$ and $C_{\infty}$ are specified in Theorem 2 (see $C_{\Pi}$ ), Proposition 2 (see $C_{t h r}$ ), and Proposition 3, respectively. 
Remark 6. Due to the boundedness of the domain we conclude from (5.6) and (5.7) the additional error bounds in the $l^{1}$-norm, i.e.,

$$
\left\|\mathcal{R}\left(\mathcal{H}_{\varepsilon} \mathbf{w}-\mathcal{H} \mathbf{w}\right)\right\|_{1} \leq \tilde{C}_{1}\left(h^{\gamma}+\varepsilon\right)
$$

for all $\mathbf{w} \in\left(\mathbb{R}^{p}\right)^{N}$ with $\|\mathcal{R} \mathbf{w}\|_{\infty} \leq C_{\infty}$ and

$$
\left\|\mathcal{R}\left(\mathbf{v}_{\varepsilon}^{0}-\mathbf{v}^{0}\right)\right\|_{1} \leq \tilde{C}_{2} \varepsilon
$$

where $\tilde{C}_{i}=(b-a) C_{i}, i=1,2$.

In addition, we will also employ some assumptions on the reference DG scheme. These are summarized in

Assumption 2 (Properties of the reference DG scheme in the mean). (i) The update step of the reference $D G$ scheme in the mean can be estimated by

$$
\|\mathcal{R H} \mathbf{w}\|_{\infty} \leq\left(1+C_{s} \tau\right)\|\mathcal{R} \mathbf{w}\|_{\infty}+C_{3} h^{\alpha}
$$

for all $\mathbf{w} \in\left(\mathbb{R}^{p}\right)^{N}$ with $\|\mathcal{R} \mathbf{w}\|_{\infty} \leq C_{\infty}$. Note that the constant $C_{s}$ only depends on the global Lipschitz bound of the source function s.

(ii) The approximate initial data converge to the initial function in the sense that

$$
\left\|\mathcal{R}\left(\mathbf{v}^{0}-\mathbf{u}^{0}\right)\right\|_{\infty} \leq C_{4} h^{\beta},
$$

where $\mathbf{u}^{0}$ denotes the coefficients corresponding to the initial function $u_{0}$.

(iii) The reference $D G$ scheme is locally $l^{1}$-stable in the mean, i.e.,

$$
\|\mathcal{R}(\mathcal{H} \mathbf{w}-\mathcal{H} \overline{\mathbf{w}})\|_{1} \leq\left(1+C_{s} \tau\right)\|\mathcal{R}(\mathbf{w}-\overline{\mathbf{w}})\|_{1}+C_{3} h^{\alpha}
$$

holds for all $\mathbf{w}, \overline{\mathbf{w}} \in\left(\mathbb{R}^{p}\right)^{N}$ with $\|\mathcal{R} \mathbf{w}\|_{\infty},\|\mathcal{R} \overline{\mathbf{w}}\|_{\infty} \leq C_{\infty}$.

In particular, the constants $C_{3}, C_{4}$ and $C_{s}$ are independent of $h, \varepsilon$.

Remark 7. Note that for the DG scheme described in Section 2 the conditions (5.10), (5.11) and (5.12) hold due to Proposition 2.1 and Lemma 2.4 in 42 .

5.2. A priori estimate for perturbation error. In order to estimate the perturbation error, we first specify the constant $C_{\infty}$ used in Assumptions 1 and 2 , Then we verify that the data of the adaptive scheme are uniformly bounded by $C_{\infty}$. With the uniform boundedness of the adaptive scheme we can then estimate the perturbation error. This will give us a rationale to choose $\varepsilon$ for a given spatial step size $h$.

Proposition 3 (Error bound for perturbation error). Let $M>0$ be arbitrary but fixed and $C_{\infty}:=e^{C_{s} T}\left(\left\|u_{0}\right\|_{L^{\infty}}+M\right)$. The ratio $\lambda=\tau / h$ is assumed to be constant and chosen small enough such that the CFL condition

$$
\lambda L_{F} \leq C F L_{\max }
$$

holds for some $0<C F L_{\max }<1$. Here $L_{F}=L_{F}\left(C_{\infty}+C_{\alpha}\right)$ denotes the Lipschitz constant of the numerical flux function for all data $\mathbf{w}$ with $\|\mathcal{R} \mathbf{w}\|_{\infty} \leq C_{\infty}$. Furthermore, let Assumptions 1 and 2 hold for the adaptive scheme and the reference $D G$ scheme, respectively.

$$
\begin{gathered}
\text { If } h \leq \min \left\{h_{\beta}, h_{\gamma}, h_{\alpha}\right\} \text { and } \varepsilon \leq \min \left\{M /\left(5 C_{2}\right), \tilde{C} h\right\} \text { with } h_{\beta}:=\left(\frac{M}{5 C_{4}}\right)^{1 / \beta} \text { and } \\
\left\{\begin{array}{lll}
h_{\gamma}:=\left(\frac{\lambda M}{5 T C_{1}}\right)^{\frac{1}{\gamma-1}}, & h_{\alpha}:=\left(\frac{\lambda M}{5 T C_{3}}\right)^{\frac{1}{\alpha-1}}, \quad \tilde{C}:=\frac{\lambda M}{5 T C_{1}}, \quad \text { if } C_{s}=0, \\
h_{\gamma}:=\left(\frac{\lambda M C_{s}}{5 C_{1}}\right)^{\frac{1}{\gamma-1}}, & h_{\alpha}:=\left(\frac{\lambda M}{5 T C_{3}}\right)^{\frac{1}{\alpha-1}}, \quad \tilde{C}:=\frac{\lambda C_{s} M}{5 C_{1}}, & \text { if } C_{s} \neq 0
\end{array}\right.
\end{gathered}
$$


for $\alpha>1$ and $\gamma>1$, then we have for all $n \leq T / \tau$

$$
\left\|\mathcal{R} \mathbf{v}_{\varepsilon}^{n}\right\|_{\infty} \leq C_{\infty}
$$

Moreover, the perturbation error can be estimated for $\tau \leq \tau_{\max }$ by

$$
\left\|\hat{v}_{\varepsilon}^{n}-\hat{v}^{n}\right\|_{L_{1}(\Omega)}=\left\|\mathcal{R}\left(\mathbf{v}_{\varepsilon}^{n}-\mathbf{v}^{n}\right)\right\|_{1} \leq C\left(h^{\gamma}+h^{\alpha}+\varepsilon\right) / \tau \quad \forall n \tau \leq T,
$$

where the constant $C$ is given by $C=2 \max \left(\tilde{C}_{1} T, C_{3} T, \tilde{C}_{2} \tau_{\max }\right)$ if $C_{s}=0$ and $C=2 e^{C_{s} T} \max \left(\tilde{C}_{1} / C_{s}, C_{3} / C_{s}, \tilde{C}_{2} \tau_{\max }\right)$ if $C_{s} \neq 0$.

Note that the constants and parameters $C_{1}, C_{2}, C_{\infty}, \gamma$ and $C_{s}, C_{3}, \beta$ stem from Assumption 1 and 2, respectively, $\tilde{C}_{1}, \tilde{C}_{2}$ are introduced in Remark 4 and $C_{\alpha}, \alpha$ correspond to the entropy enforcing conditions (2.12), (2.13) of the limiter (2.10).

Remark 8. The bound $C_{\infty}$ contains an upper bound $e^{C_{s} T}\left\|u_{0}\right\|_{L^{\infty}}$ for the exact solution that is enlarged by a constant $M$ to allow for numerical errors. The constant $M$ can be chosen arbitrarily small, resulting in possibly very small mesh size $h$ and small values of $\varepsilon$.

Proof of Proposition 3, First of all we show (5.14) by induction. For $n=0$ we conclude that

$$
\begin{aligned}
\left\|\mathcal{R} \mathbf{v}_{\varepsilon}^{0}\right\|_{\infty} \leq\left\|\mathcal{R}\left(\mathbf{v}_{\varepsilon}^{0}-\mathbf{v}^{0}\right)\right\|_{\infty}+\left\|\mathcal{R}\left(\mathbf{v}^{0}-\mathbf{u}^{0}\right)\right\|_{\infty}+\left\|\mathcal{R} \mathbf{u}^{0}\right\|_{\infty} \\
\quad \leq C_{2} \varepsilon+C_{4} h^{\beta}+\left\|u_{0}\right\|_{L^{\infty}} \leq \frac{M}{5}+\frac{M}{5}+\left\|u_{0}\right\|_{L^{\infty}} \leq C_{\infty}
\end{aligned}
$$

where we use the bounds on $\varepsilon$ and $h$. Now we assume that $\left\|\mathcal{R} \mathbf{v}_{\varepsilon}^{j}\right\|_{\infty} \leq C_{\infty}$ for $j=0, \ldots, n-1$. Then we estimate the mean values of the adaptive scheme by

$$
\begin{aligned}
\left\|\mathcal{R} \mathbf{v}_{\varepsilon}^{n}\right\|_{\infty} & =\left\|\mathcal{R} \mathcal{H}_{\varepsilon} \mathbf{v}_{\varepsilon}^{n-1}\right\|_{\infty} \\
& \leq\left\|\mathcal{R}\left(\mathcal{H}_{\varepsilon} \mathbf{v}_{\varepsilon}^{n-1}-\mathcal{H} \mathbf{v}_{\varepsilon}^{n-1}\right)\right\|_{\infty}+\left\|\mathcal{R} \mathcal{H} \mathbf{v}_{\varepsilon}^{n-1}\right\|_{\infty} \\
& \leq C_{1}\left(h^{\gamma}+\varepsilon\right)+\left(1+C_{s} \tau\right)\left\|\mathcal{R} \mathbf{v}_{\varepsilon}^{n-1}\right\|_{\infty}+C_{3} h^{\alpha}
\end{aligned}
$$

and then infer by repeating this estimate that

$$
\left\|\mathcal{R} \mathbf{v}_{\varepsilon}^{n}\right\|_{\infty} \leq\left(1+C_{s} \tau\right)^{n}\left\|\mathcal{R} \mathbf{v}_{\varepsilon}^{0}\right\|_{\infty}+\left(C_{1} h^{\gamma}+C_{1} \varepsilon+C_{3} h^{\alpha}\right) \sum_{j=0}^{n-1}\left(1+C_{s} \tau\right)^{j}
$$

We now apply (5.16). By the bounds on $h$ and $\varepsilon$ we then estimate further, where we distinguish two cases. If $C_{s}=0$, then $n \leq T / \tau=T /(\lambda h)$ and we obtain

$$
\begin{aligned}
\left\|\mathcal{R} \mathbf{v}_{\varepsilon}^{n}\right\|_{\infty} & \leq\left\|u_{0}\right\|_{L^{\infty}}+\frac{2 M}{5}+\frac{T}{\lambda}\left(C_{1} h^{\gamma-1}+C_{1} \frac{\varepsilon}{h}+C_{3} h^{\alpha-1}\right) \\
& \leq\left\|u_{0}\right\|_{L^{\infty}}+2 M / 5+3 M / 5=C_{\infty} .
\end{aligned}
$$

Otherwise, we bound the geometric sum by $e^{C_{s} T} /\left(C_{s} \tau\right)$ with $\tau=\lambda h$ and then estimate

$$
\begin{aligned}
\left\|\mathcal{R} \mathbf{v}_{\varepsilon}^{n}\right\|_{\infty} & \leq e^{C_{s} T}\left(\left\|u_{0}\right\|_{L^{\infty}}+2 M / 5+\left(C_{1} h^{\gamma-1}+C_{1} \frac{\varepsilon}{h}+C_{3} h^{\alpha-1}\right) /\left(C_{s} \lambda\right)\right) \\
& \leq e^{C_{s} T}\left(\left\|u_{0}\right\|_{L^{\infty}}+2 M / 5+3 M / 5\right)=C_{\infty}
\end{aligned}
$$

Next we verify (5.15). First of all we note by (5.10) that the reference scheme is uniformly bounded, i.e., there exists a constant $C_{\infty}=C_{\infty}\left(T, u_{0}\right)$ with $\left\|\mathcal{R} \mathbf{v}^{n}\right\|_{\infty} \leq$ 
$C_{\infty}$ for all $n \leq \frac{T}{\tau}$. Then we see by the assumptions that

$$
\begin{aligned}
\left\|\mathcal{R}\left(\mathbf{v}_{\varepsilon}^{n}-\mathbf{v}^{n}\right)\right\|_{1} & =\left\|\mathcal{R}\left(\mathcal{H}_{\varepsilon} \mathbf{v}_{\varepsilon}^{n-1}-\mathcal{H} \mathbf{v}_{\varepsilon}^{n-1}\right)\right\|_{1}+\left\|\mathcal{R}\left(\mathcal{H} \mathbf{v}_{\varepsilon}^{n-1}-\mathcal{H} \mathbf{v}^{n-1}\right)\right\|_{1} \\
& \leq \tilde{C}_{1}\left(h^{\gamma}+\varepsilon\right)+\left(1+C_{s} \tau\right)\left\|\mathcal{R}\left(\mathbf{v}_{\varepsilon}^{n-1}-\mathbf{v}^{n-1}\right)\right\|_{1}+C_{3} h^{\alpha}
\end{aligned}
$$

Repeating this argument we obtain

$$
\left\|\mathcal{R}\left(\mathbf{v}_{\varepsilon}^{n}-\mathbf{v}^{n}\right)\right\|_{1} \leq\left(1+C_{s} \tau\right)^{n}\left\|\mathcal{R}\left(\mathbf{v}_{\varepsilon}^{0}-\mathbf{v}^{0}\right)\right\|_{1}+\left(\tilde{C}_{1}\left(h^{\gamma}+\varepsilon\right)+C_{3} h^{\alpha}\right) \sum_{j=0}^{n-1}\left(1+C_{s} \tau\right) .
$$

In case of $C_{s} \neq 0$ we conclude that

$$
\begin{aligned}
\left\|\mathcal{R}\left(\mathbf{v}_{\varepsilon}^{n}-\mathbf{v}^{n}\right)\right\|_{1} & \leq \tilde{C}_{2} \varepsilon+T / \tau\left(\tilde{C}_{1}\left(h^{\gamma}+\varepsilon\right)+C_{3} h^{\alpha}\right) \\
& \leq 2 \max \left(\tilde{C}_{1} T, C_{3} T, \tilde{C}_{2} \tau_{\max }\right)\left(h^{\gamma}+h^{\alpha}+\varepsilon\right) / \tau ;
\end{aligned}
$$

otherwise we obtain

$$
\begin{aligned}
\left\|\mathcal{R}\left(\mathbf{v}_{\varepsilon}^{n}-\mathbf{v}^{n}\right)\right\|_{1} & \leq e^{C_{s} T}\left(\tilde{C}_{2} \varepsilon+\frac{1}{C_{s} \tau}\left(\tilde{C}_{1}\left(h^{\gamma}+\varepsilon\right)+C_{3} h^{\alpha}\right)\right) \\
& \leq 2 e^{C_{s} T} \max \left(\tilde{C}_{1} / C_{s}, C_{3} / C_{s}, \tilde{C}_{2} \tau_{\max }\right)\left(h^{\gamma}+h^{\alpha}+\varepsilon\right) / \tau
\end{aligned}
$$

Due to the choice of the constant the assertion holds.

Because of estimate (5.15) we may now balance the discretization error of the reference scheme and the perturbation error. This gives an "optimal" choice for the threshold value.

Corollary 1 (Choice of the threshold parameter). If the discretization error of the reference $D G$ scheme is bounded by $\|\hat{\eta}(t, \cdot)\|_{L^{1}(\Omega)} \leq C h^{\beta}$ for some $\beta>0$ and $\alpha \geq 1+\beta$ in the entropy enforcing bound (2.12) and $\gamma \geq 1+\beta$ in (5.6), then the accuracy of the reference scheme is preserved by the adaptive DG scheme provided that $\varepsilon \sim h^{1+\beta}$ and the time step $\tau$ is limited by a CFL condition.

Since the error bounds in Proposition 3 are worst-case estimates, the choice $\varepsilon \sim h^{1+\beta}$ might be too pessimistic. The numerical studies in Section 7 indicate that $\varepsilon \sim h$ seems to be sufficient to ensure efficient and accurate solutions.

5.3. Error bound with Runge-Kutta time stepping. In the previous sections we considered the step operator $\mathcal{H}$ to be the explicit Euler step of the reference DG scheme. However, in view of a higher order method in time, we are interested in analyzing the adaptive version of the Runge-Kutta time step operator $\mathcal{H}^{R K}$.

Let the adaptive scheme with Runge-Kutta time stepping be defined by

$$
\mathbf{u}_{\varepsilon}^{(0)}=\mathbf{w}, \quad \mathbf{u}_{\varepsilon}^{(s)}=\sum_{j=0}^{s-1} \alpha_{s, j} \mathcal{H}_{\varepsilon}^{\tau_{s, j}} \mathbf{u}_{\varepsilon}^{(j)}, \quad s=1, \ldots, m, \quad \mathcal{H}_{\varepsilon}^{R K} \mathbf{w}=\mathbf{u}_{\varepsilon}^{(m)},
$$

where $\mathcal{H}_{\varepsilon}^{\tau_{s, j}}$ denotes one step of the adaptive scheme with time step size $\tau_{s, j}$. The coefficients are assumed to satisfy the consistency condition $\sum_{j=0}^{s-1} \alpha_{s, j}=1$.

For equation (5.15) in Proposition 3 to hold, we have to show the uniform boundedness of the adaptive Runge-Kutta scheme, i.e.,

$$
\left\|\mathcal{R} \mathbf{v}_{\varepsilon}^{R K, n}\right\|_{\infty} \leq C_{\infty}
$$


with $\mathbf{v}_{\varepsilon}^{R K, n}=\mathcal{H}_{\varepsilon}^{R K} \mathbf{v}_{\varepsilon}^{R K, n-1}$ and $\mathbf{v}_{\varepsilon}^{R K, 0}=\mathbf{v}_{\varepsilon}^{0}$, as well as the boundedness of the update error, i.e.,

$$
\left\|\mathcal{R}\left(\mathcal{H}_{\varepsilon}^{R K} \mathbf{w}-\mathcal{H}^{R K} \mathbf{w}\right)\right\|_{1} \leq C_{R K}\left(\bar{C}_{1}\left(h^{\gamma}+\varepsilon\right)+C_{p} h^{\alpha}\right) .
$$

The local $l^{1}$-stability of the reference Runge-Kutta scheme follows from Lemma 2.6 in [42. To show the boundedness of the update error, we use the same argument as in the proof of estimate (5.14) of Proposition 3, where we replace (5.17) by

$$
\left\|\mathcal{R} \mathbf{v}_{\varepsilon}^{R K, n}\right\|_{\infty} \leq\left(1+C_{R K} C_{s} \tau\right)\left\|\mathcal{R} \mathbf{v}_{\varepsilon}^{R K, n-1}\right\|_{\infty}+C_{R K} C_{1}\left(h^{\gamma}+\varepsilon\right)+C_{R K} C_{3} h^{\alpha} .
$$

Here $C_{R K}$ is some constant depending on the global Lipschitz constant $L_{s}$ of the source function, $m$ the number of stages and $\tau_{\max }$ a maximal time step size. For details see [42. To see this, we show by induction that

$$
\left\|\mathcal{R} \mathbf{u}_{\varepsilon}^{(s)}\right\|_{\infty} \leq\left(1+L_{s} \tau\right)^{s}\left\|\mathcal{R} \mathbf{u}_{\varepsilon}^{(0)}\right\|_{\infty}+\sum_{r=0}^{s-1}\left(1+L_{s} \tau\right)^{r}\left(C_{1}\left(\varepsilon+h^{\gamma}\right)+C_{p} h^{\alpha}\right),
$$

and then use the fact that for some constant $C_{1}$ and $\tau \leq \tau_{\max }$ we can estimate $\left(1+C_{1} \tau\right)^{s} \leq 1+C 1 \tau C 2$ with $C_{2}=C_{2}(m)$. Then all steps in the proof of the estimate (5.14) in Proposition 3 hold true.

To show (5.21) we similarly use the local $l^{1}$-stability of the explicit Euler scheme; see Lemma 2.4 in [42, and (5.8). Then we finally can conclude:

Conclusion 1. Let the assumptions of Proposition 3 hold. The Runge-Kutta perturbation error is uniformly bounded, i.e.,

$$
\left\|\mathcal{R}\left(\mathbf{v}_{\varepsilon}^{R K, n}-\mathbf{v}^{R K, n}\right)\right\|_{1} \leq C\left(h^{\gamma}+h^{\alpha}+\varepsilon\right) / \tau \text { for all } n \tau \leq T .
$$

Here $\gamma$ and $\alpha$ stem from Assumption 1 and the entropy enforcing conditions (2.12), (2.13) of the limiter (2.10), respectively.

\section{Adaptive MR-DG scheme: Verification of Sufficient Conditions}

Since the adaptive MR-DG scheme determined by Algorithm 5 only acts on the local single-scale coefficients $\mathbf{v}_{\mathcal{G}}$ corresponding to an adaptive grid characterized by $\mathcal{G}$ but the analysis is to be performed for the single-scale coefficients $\mathbf{v}_{L}$ on the highest refinement level, we have to rewrite the algorithm on the reference mesh. For this purpose, we introduce the projection operator $\mathcal{P}_{\mathcal{G}}:\left(\mathbb{R}^{p}\right)^{\# \mathcal{G}} \rightarrow\left(\mathbb{R}^{p}\right)^{N_{L}}$ that maps the local single-scale coefficients $\mathbf{v}_{\mathcal{G}}=\left(v_{l, k, i}\right)_{(l, k) \in \mathcal{G}, i \in \mathcal{P}}$ to the reference mesh by successively applying (3.15) with $d_{l, k, j}=0, j \in \mathcal{P}$, to the subcells of cells $V_{l, k}$, $(l, k) \in \mathcal{G}$. To reverse the projection step we also need the restriction operator $\mathcal{R}_{\mathcal{G}}:\left(\mathbb{R}^{p}\right)^{N_{L}} \rightarrow\left(\mathbb{R}^{p}\right)^{\# \mathcal{G}}$ by which we map the single-scale coefficients $\mathbf{v}_{L}$ to the local single-scale coefficients $\mathbf{v}_{\mathcal{G}}$ by successively agglomerating cells applying (3.14) (a) from fine-scale down to cells $V_{l, k},(l, k) \in \mathcal{G}$. Note that $\mathcal{R}_{\mathcal{G}} \mathcal{P}_{\mathcal{G}}=I$, but in general $\mathcal{P}_{\mathcal{G}} \mathcal{R}_{\mathcal{G}} \neq I$.

Then we map each step of Algorithm 5 to the reference mesh:

(0) The initial data are $\mathbf{v}_{L}^{n}:=\mathcal{P}_{\mathcal{G}^{n}} \mathbf{v}_{\mathcal{G}^{n}}$.

(1) The refinement step does not change the single-scale coefficients because of Step 0 in Algorithm 3, i.e., $\tilde{\mathbf{v}}_{L}^{n}:=\mathcal{P}_{\tilde{\mathcal{G}}^{n+1}} \mathbf{v}_{\tilde{\mathcal{G}}^{n+1}}=\mathcal{P}_{\mathcal{G}^{n}} \mathbf{v}_{\mathcal{G}^{n}}=\mathbf{v}_{L}^{n}$.

(2a) The limiter from Algorithm 4 only acts on higher order coefficients on the highest resolution level, i.e., $\overline{\mathbf{v}}_{L}^{n}:=\mathcal{P}_{\tilde{\mathcal{G}}^{n+1}} \overline{\mathbf{v}}_{\tilde{\mathcal{G}}^{n+1}}=\Pi_{L, \tilde{\mathcal{G}}^{n+1}} \mathbf{v}_{L}^{n}$. Here $\Pi_{L, \mathcal{G}}:\left(\mathbb{R}^{p}\right)^{N_{L}} \rightarrow\left(\mathbb{R}^{p}\right)^{N_{L}}$ denotes the limiter operator that only modifies 
the data in cells $(L, K) \in \mathcal{G}$ of the adaptive grid $\mathcal{G}$, where both neighbors are also contained in $\mathcal{G}$ by applying the reference limiter (2.10).

(2b) Let $\mathcal{E}_{\mathcal{G}}:\left(\mathbb{R}^{p}\right)^{\# \mathcal{G}} \rightarrow\left(\mathbb{R}^{p}\right)^{\# \mathcal{G}}$ denote the local evolution operator on the adaptive grid $\mathcal{G}$ by which we perform the time evolution for the local singlescale coefficients in Step 2b of Algorithm 5. Then the evolution step can be mapped to the reference mesh by $\tilde{\mathbf{v}}_{L}^{n+1}:=\mathcal{P}_{\tilde{\mathcal{G}}^{n+1}} \tilde{\mathbf{v}}_{\tilde{\mathcal{G}}^{n+1}}=\mathcal{E}_{L, \tilde{\mathcal{G}}^{n+1}} \overline{\mathbf{v}}_{L}^{n}$. Here the operator $\mathcal{E}_{L, \tilde{\mathcal{G}}}:\left(\mathbb{R}^{p}\right)^{N_{L}} \rightarrow\left(\mathbb{R}^{p}\right)^{N_{L}}$ is defined by $\mathcal{E}_{L, \mathcal{G}}:=\mathcal{P}_{\mathcal{G}} \mathcal{E}_{\mathcal{G}} \mathcal{R}_{\mathcal{G}}$.

(3) The coarsening step is realized by performing hard thresholding on the detail coefficients. For this purpose we can employ the threshold operator $\mathcal{A}_{\varepsilon}$ defined in (3.21), i.e., $\mathbf{v}_{L}^{n+1}:=\mathcal{P}_{\mathcal{G}^{n+1}} \mathbf{v}_{\mathcal{G}^{n+1}}=\mathcal{A}_{\varepsilon} \tilde{\mathbf{v}}_{L}^{n+1}$.

Putting all these steps together we finally end up with the adaptive evolution operator

$$
\mathcal{H}_{\varepsilon} \mathbf{w}:=\mathcal{A}_{\varepsilon} \mathcal{E}_{L, \tilde{\mathcal{G}}(\mathbf{w})} \Pi_{L, \tilde{\mathcal{G}}(\mathbf{w})} \mathbf{w} \quad \forall \mathbf{w} \in\left(\mathbb{R}^{p}\right)^{N_{L}}
$$

where $\tilde{\mathcal{G}}(\mathbf{w})$ characterizes the adaptive grid determined by the prediction set $\tilde{\mathcal{D}}(\mathbf{w})$ according to Remark 2 .

In the course of the error analysis for the adaptive MR-DG scheme presented in Section 4 we now have to verify (5.6) and (5.7) of Assumption 1 . Then the adaptive evolution operator $\mathcal{H}_{\varepsilon}$ in comparison with the reference DG evolution operator $\mathcal{H} \mathbf{w}=\mathcal{E}_{L} \Pi_{L} \mathbf{w}$ only introduces an error bounded by $\varepsilon$, i.e., Proposition 3 holds true for our scheme. Here the index $L$ emphasizes the fact that the reference scheme performs limiting and evolution on the uniform reference grid on level $L$.

For that we will prove for any data $\mathbf{w} \in\left(\mathbb{R}^{p}\right)^{N_{L}}$ the following two conditions on the limiting process

$$
\left\|\mathcal{R}\left(\mathcal{E}_{L} \Pi_{L, \tilde{\mathcal{G}}(\mathbf{w})} \mathbf{w}-\mathcal{E}_{L} \Pi_{L} \mathbf{w}\right)\right\|_{\infty} \leq C_{\Pi} \varepsilon
$$

and on the evolution process

$$
\left\|\mathcal{R}\left(\mathcal{E}_{L, \tilde{\mathcal{G}}(\mathbf{w})} \Pi_{L, \tilde{\mathcal{G}}(\mathbf{w})} \mathbf{w}-\mathcal{E}_{L} \Pi_{L, \tilde{\mathcal{G}}(\mathbf{w})} \mathbf{w}\right)\right\|_{\infty} \leq C_{\mathcal{E}} \varepsilon .
$$

Note that the constants $C_{\Pi}$ and $C_{\mathcal{E}}$ depend on $\|\mathcal{R} \mathrm{w}\|+\varepsilon+h_{L}^{\alpha}$. These conditions are linked to the reliability of the prediction and the limiting process in the sense of (4.13). Finally, we will conclude that the following theorem holds.

Theorem 1 (Error estimate of perturbation error). Let Assumption 2 hold true for the reference $D G$ scheme. The discretization is chosen such that the CFL condition (5.13) holds. In particular, the spatial discretization $h_{L}$ and the threshold value $\varepsilon$ are bounded from above. We initialize the adaptive scheme by

$$
\mathbf{v}_{\varepsilon}^{0}=\mathcal{A}_{\varepsilon} \mathbf{v}^{0},
$$

where $\mathcal{A}_{\varepsilon}$ is the approximation operator defined by (3.21). Then conditions (5.6) and (5.7) of Assumption 1 hold true for the adaptive MR-DG scheme described in Algorithm 5. Moreover the perturbation error (5.5) can be estimated by (5.15).

This is the main result of the paper. A proof is given at the end of this section.

6.1. Properties of the prediction set. Before we can verify the conditions (E) and $(\mathrm{L})$, we need to summarize some properties that can be concluded from the prediction and the limiting strategy determined by Algorithms 3 and 4 , respectively. 
Lemma 1 (Properties of the prediction set). Let $\tilde{\mathcal{D}}(\mathbf{w})$ be the prediction set determined by the data $\mathbf{w} \in\left(\mathbb{R}^{p}\right)^{N_{L}}$. Then the following estimates hold for the function $v_{L}$ corresponding to the coefficients $\mathbf{w}$ on any cell $V_{l, k}$ with $(l, k, i) \notin \tilde{\mathcal{D}}(\mathbf{w})$ and $l<L$ for all $i \in \mathcal{P}$ :

- The prediction does not change the data on the reference mesh.

- The function $v_{L}$ is a polynomial on $V_{l, k}$, i.e.,

$$
\left.v_{L}\right|_{V_{l, k}} \in \Pi_{p-1} \text {. }
$$

- Reliability of evolution step:

$$
\begin{gathered}
\left|v_{L}\left(x^{+}\right)-v_{L}\left(x^{-}\right)\right| \leq \varepsilon, \quad x=x_{l, k} \text { or } x=x_{l, k+1}, \\
\frac{\left(h_{l} / 2\right)^{p}}{p !}\left|\frac{d^{p+1}}{d x^{p+1}} f\left(v_{L}(x)\right)\right| \leq 2^{l} \varepsilon \quad \forall x \in V_{l, k}, \\
\frac{\left(h_{l} / 2\right)^{p}}{p !}\left|\frac{d^{p}}{d x^{p}} s\left(v_{L}(x)\right)\right| \leq 2^{l} \varepsilon \quad \forall x \in V_{l, k} .
\end{gathered}
$$

- Reliability of limiter process:

- At cell interfaces:

$$
\begin{aligned}
& c_{L, k^{+}}^{ \pm}=1 \vee\left(\left|v_{L}\left(x_{l, k}^{+}\right)-v_{L, k^{+}, 0}\right| \leq \varepsilon \wedge\left|v_{L}\left(x_{L, k^{+}+1}^{-}\right)-v_{L, k^{+}, 0}\right| \leq \varepsilon\right), \\
& c_{L, k-}^{ \pm}=1 \vee\left(\left|v_{L}\left(x_{l, k+1}^{-}\right)-v_{L, k^{-}, 0}\right| \leq \varepsilon \wedge\left|v_{L}\left(x_{L, k^{-}}^{+}\right)-v_{L, k^{-}, 0}\right| \leq \varepsilon\right)
\end{aligned}
$$

with $k^{+}=2^{(L-l)} k$ and $k^{-}=2^{(L-l)}(k+1)-1$ and the notation from (2.11).

- Bounded curvature on cell:

$$
\left|v_{L}^{\prime \prime}(x)\right| \leq C_{L} \frac{\varepsilon}{h_{L}^{2}} \text { holds for all } x \in V_{l, k}
$$

with a fixed constant $C_{L}>0$.

- Entropy bound

$$
\left|v_{L}^{\prime}(x)\right| \leq C_{\alpha} h_{L}^{\alpha-1} \quad \forall x \in V_{l, k},
$$

where $C_{\alpha}, \alpha$ stem from the entropy enforcing conditions (2.12), (2.13) of the limiter (2.10).

- Buffer zone to cells on lower levels:

If for the grid $\tilde{\mathcal{G}}(\mathbf{w})$ corresponding to $\tilde{\mathcal{D}}(\mathbf{w})$ the index $(L, k) \in \tilde{\mathcal{G}}(\mathbf{w})$ with

$(L, k-1) \notin \tilde{\mathcal{G}}(\mathbf{w})$ or $(L, k+1) \notin \tilde{\mathcal{G}}(\mathbf{w})$, then (4) with $k^{ \pm}=k$ and (L6) must hold for the index $(L, k)$ as well.

Proof. The properties follow by the construction of the prediction set, because in Algorithm 3 we locally refine in each Step * the adaptive grid until either the corresponding estimate $\left(^{*}\right)$ holds for some $l^{\prime}<L$ or we arrive at the highest level $l^{\prime}=L$.

It is worthwhile mentioning that property (P0) follows from enforcing the tree property (3.23); see Step 8 in Algorithm 3. Then there are no detail coefficients corresponding to cells on higher levels contained in the cell $V_{l, k}$, and, hence, $v_{L}$ is a polynomial on that cell. 
To prove estimate (E2) we note that by Step E2 we enforce the inequality

$$
\left(h_{l} / 2\right)^{p} \sum_{\mathbf{k} \in T_{p+1}} \frac{p+1}{k_{1} ! \cdots k_{p+1} !} D_{f}^{|\mathbf{k}|} \prod_{j=1, k_{j} \geq 1}^{p+1}\left(\frac{D_{v}^{j}}{j !}\right)^{k_{j}} \leq 2^{l} \varepsilon .
$$

Note that due to dyadic grid refinement $h_{l}=2^{-l} h_{0}$. We proceed similarly as in the case of estimate (E3).

Remark 9. In order to verify condition (E), we need that the estimates (E1)-(E3) still hold after limiting, i.e., they remain true with $\mathbf{v}$ replaced by $\Pi_{L, \tilde{\mathcal{G}}^{n}} \mathbf{v}$. For this reason we inflate the prediction set in the neighborhood of cells located on the highest refinement level $L$; otherwise the limiter could alter the point value of the data from outside of (E1); this is enforced by (B7).

Remark 10. The estimates ( $\underline{\mathrm{L} 4}$ ) and (L5) ensure that in regions where the reference limiter modifies the data on level $L$ significantly, we resolve these modifications. Otherwise the error introduced by the adaptive limiter is bounded. Furthermore, the estimate ([-6) is needed to enforce convergence to the entropy solution. Note that the conditions (L4)-( $(\mathrm{L} 6)$ are adjusted to the reference limiter (2.10).

In order to analyze the influence of the adaptive limiter in Algorithm 4, we need properties under which the reference limiter (2.10) does not change the data.

Lemma 2 (Inactivity of reference limiter). Let $\mathbf{v} \in\left(\mathbb{R}^{p}\right)^{N}$ with its corresponding function $v \in S^{p}$. Consider a cell $V_{k}$. If the estimates

$$
\begin{gathered}
0 \leq \frac{\bar{v}_{k}^{+}}{v_{k, 0}-v_{k-1,0}} \leq 1, \quad 0 \leq \frac{\bar{v}_{k}^{-}}{v_{k+1,0}-v_{k, 0}} \leq 1, \\
0 \leq \frac{\bar{v}_{k}^{+}}{v_{k+1,0}-v_{k, 0}} \leq 1, \quad 0 \leq \frac{\bar{v}_{k}^{-}}{v_{k, 0}-v_{k-1,0}} \leq 1, \\
\max _{x \in V_{k}}\left|v^{\prime}(x)\right| \leq C_{\alpha} h^{\alpha-1}
\end{gathered}
$$

hold with $C_{\alpha}, \alpha$ according to (2.12), (2.13), then the limiter (2.10) does not change the data, i.e., $\tilde{v}_{k, i}=v_{k, i}$ for all $i \in \mathcal{P}$.

This lemma also holds true for both the van Leer limiter and the Shu limiter. A proof can be found in [42, p. $10 \mathrm{ff}$.

To analyze the influence of the adaptive limiter on the finest refinement level, we show the following lemma:

Lemma 3 (Influence of limiter on finest scale). Let $\mathbf{v} \in\left(\mathbb{R}^{p}\right)^{N}$ such that $v$ is a polynomial on $I:=V_{L, k-1} \cup V_{L, k} \cup V_{L, k+1}$ and let the bounds

$$
\left|v^{\prime \prime}(x)\right| \leq C_{L} \frac{\varepsilon}{h_{L}^{2}} \quad \text { for all } x \in I \quad \text { and } \quad \max _{x \in V_{k}}\left|v^{\prime}(x)\right| \leq C_{\alpha} h_{L}^{\alpha-1}
$$

hold with $C_{\alpha}, \alpha$ according to (2.12), (2.13). Then, either $c_{L, k}^{ \pm}=1$, i.e., the reference limiter does not change the data, or

$$
\left|v\left(x_{L, k+1}\right)-v_{L, k, 0}\right| \leq \bar{C}_{L} \varepsilon \quad \text { and } \quad\left|v\left(x_{L, k}\right)-v_{L, k, 0}\right| \leq \bar{C}_{L} \varepsilon
$$

with $\bar{C}_{L}=12 C_{L}$. 
Proof. We distinguish two cases for polynomials with small and large gradient, respectively.

Case 1 (There exists $z \in I$ with $\left.\left|v^{\prime}(z)\right|<4 C_{L} \frac{\varepsilon}{h_{L}}\right)$. For any $x \in I$ we have by the Taylor expansion

$$
v(x)=v(z)+(x-z) v^{\prime}(z)+\int_{z}^{x}(x-\xi) v^{\prime \prime}(\xi) d \xi .
$$

Let $\bar{x}_{k} \in V_{L, k}$ denote a point with $v\left(\bar{x}_{k}\right)=v_{L, k, 0}$; it exists by the intermediate value theorem. Using the expansion twice for $\bar{x}_{k}$ and $x_{L, k+1}$ we obtain

$$
\begin{aligned}
& \left|v\left(x_{L, k+1}\right)-v_{L, k, 0}\right| \\
& =\left|\left(x_{L, k+1}-\bar{x}_{k}\right) v^{\prime}(z)+\int_{z}^{x_{L, k+1}}\left(x_{L, k+1}-\xi\right) v^{\prime \prime}(\xi) d \xi-\int_{z}^{\bar{x}_{k}}\left(\bar{x}_{k}-\xi\right) v^{\prime \prime}(\xi) d \xi\right| \\
& \leq h_{L}\left|v^{\prime}(z)\right|+2 h_{L} 2 h_{L}\left\|v^{\prime \prime}\right\|_{L^{\infty}(I)}+2 h_{L} 2 h_{L}\left\|v^{\prime \prime}\right\|_{L^{\infty}(I)} \leq 12 C_{L} \varepsilon .
\end{aligned}
$$

Analogously we conclude $\left|v\left(x_{L, k}\right)-v_{L, k, 0}\right| \leq 12 C_{L} \varepsilon$.

Case $2\left(\left|v^{\prime}(x)\right| \geq 4 C_{L} \frac{\varepsilon}{h_{L}}\right.$ for all $\left.x \in I\right)$. Without loss of generality, we assume $v^{\prime}>0$ in the second case, otherwise we consider $-v$. It remains to show that for $v^{\prime}(x) \geq 4 C_{L} \frac{\varepsilon}{h_{L}}$ for all $x \in I$ the estimates 6.3a and a.3b hold. From $v^{\prime}>0$ and $v_{L, k^{\prime}, 0}=v\left(\bar{x}_{k^{\prime}}\right)$ for some $\bar{x}_{k^{\prime}} \in V_{L, k^{\prime}}$ we immediately conclude that

$v_{L, k, 0}-v_{L, k-1,0}=v\left(\bar{x}_{k}\right)-v\left(\bar{x}_{k-1}\right) \geq 0, \quad v_{L, k+1,0}-v_{L, k, 0}=v\left(\bar{x}_{k+1}\right)-v\left(\bar{x}_{k}\right) \geq 0$, $v\left(x_{L, k+1}\right)-v_{L, k, 0}=v\left(x_{L, k+1}\right)-v\left(\bar{x}_{k}\right) \geq 0, \quad v_{L, k, 0}-v\left(x_{L, k}\right)=v\left(\bar{x}_{k}\right)-v\left(x_{L, k}\right) \geq 0$.

These are the bounds from below in (6.3a) and (6.3b). Additionally, we have the bounds

$$
v_{L, k, 0}-v\left(x_{L, k}\right) \leq v_{L, k, 0}-v_{L, k-1,0}, \quad v\left(x_{L, k+1}\right)-v_{L, k, 0} \leq v_{L, k+1,0}-v_{L, k, 0}
$$

and conclude the upper bounds in (6.3a).

It remains to show the upper bounds in (6.3b), i.e., we must show

$$
v_{L, k, 0}-v\left(x_{L, k}\right) \leq v_{L, k+1,0}-v_{L, k, 0} \quad \text { and } \quad v\left(x_{L, k+1}\right)-v_{L, k, 0} \leq v_{L, k, 0}-v_{L, k-1,0} .
$$

Here we confine ourselves to the second one. For that let $g:=\min _{x \in I} v^{\prime}(x)$ and $x_{g}$ be a point such that $v^{\prime}\left(x_{g}\right)=g$. We will show

$$
\text { a) } v_{L, k, 0}-v_{L, k-1,0} \geq g h_{L} \text { and b) } v\left(x_{L, k+1}\right)-v_{L, k, 0} \leq g h_{L} \text {. }
$$

Note that for any $x, x_{0} \in I$ we have by definition of $g$ $v(x) \geq v\left(x_{0}\right)+\left(x-x_{0}\right) g, \quad$ for $x \geq x_{0}, \quad$ and $\quad v(x) \leq v\left(x_{0}\right)+\left(x-x_{0}\right) g, \quad$ for $x \leq x_{0}$, and therefore

$$
v_{L, k, 0}=\frac{1}{h_{L}} \int_{x_{L, k}}^{x_{L, k+1}} v(x) d x \geq \frac{1}{h_{L}} \int_{x_{L, k}}^{x_{L, k+1}} v\left(x_{L, k}\right)+\left(x-x_{L, k}\right) g d x=v\left(x_{L, k}\right)+g \frac{h_{L}}{2},
$$

and similarly $v_{L, k-1,0} \leq v\left(x_{L, k}\right)-g \frac{h_{L}}{2}$. Subtracting these terms we conclude (6.5) $)$.

To show (6.5) we expand $v$ around $x_{L, k+1}$, i.e.,

$$
v(x)=v\left(x_{L, k+1}\right)+\left(x-x_{L, k+1}\right) v^{\prime}\left(x_{L, k+1}\right)+\frac{\left(x-x_{L, k+1}\right)^{2}}{2} v^{\prime \prime}(\xi)
$$


for some $\xi \in\left[x, x_{L, k+1}\right]$. Note that $v^{\prime}\left(x_{L, k+1}\right)=v^{\prime}\left(x_{g}\right)+\int_{x_{g}}^{x_{L, k+1}} v^{\prime \prime}(y) d y$. This yields

$$
\begin{aligned}
& v\left(x_{L, k+1}\right)-v_{L, k, 0} \\
& =\frac{1}{2} h_{L} g+\frac{1}{2} h_{L} \int_{x_{g}}^{x_{L, k+1}} v^{\prime \prime}(y) d y-\frac{1}{2 h_{L}} \int_{x_{L, k}}^{x_{L, k+1}}\left(x-x_{L, k+1}\right)^{2} v^{\prime \prime}(\xi(x)) d x \\
& \leq \frac{1}{2} h_{L} g+\frac{1}{2} h_{L} 2 h_{L}\left\|v^{\prime \prime}\right\|_{L^{\infty}(I)}+\frac{1}{2 h_{L}} h_{L} h_{L}^{2}\left\|v^{\prime \prime}\right\|_{L^{\infty}(I)} \leq \frac{1}{2} h_{L} g+2 h_{L}^{2} C_{L} \frac{\varepsilon}{h_{L}^{2}}=g h_{L},
\end{aligned}
$$

where we bound the integrals by the size of the integration domain together with the maximal absolute value of the integrand and use the bound on $v^{\prime \prime}$.

From Lemma 3 and (L4) we finally conclude that

Corollary 2. Let $V_{l, k}$ be a cell of the adaptive grid, i.e., $(l, k) \in \tilde{\mathcal{G}}$, and $l<L$. Then, for each cell of the uniform grid $V_{L, k^{\prime}} \subset V_{l, k}$ we have either $c_{L, k^{\prime}}^{ \pm}=1$ or

$$
\left|v\left(x_{L, k^{\prime}}^{+}\right)-v_{L, k^{\prime}, 0}\right| \leq \bar{C}_{L} \varepsilon \text { and }\left|v\left(x_{L, k^{\prime}+1}^{-}\right)-v_{L, k^{\prime}, 0}\right| \leq \bar{C}_{L} \varepsilon,
$$

with $\bar{C}_{L}$ according to Lemma 3. In both cases we have

$$
\left|v^{\prime}(x)\right| \leq C_{\alpha} h_{L}^{\alpha-1}
$$

with $C_{\alpha}, \alpha$ according to (2.12), (2.13).

Proof. If the neighboring cells are inside the coarse grid cell as well, i.e., $V_{L, k^{\prime} \pm 1} \subset$ $V_{l, k}$, then we use Lemma 3] otherwise the assertion holds by (L4).

Corollary 3. Let $V_{L, k}$ be a cell of the uniform grid. Then, either the adaptive limiter $\Pi_{L, \tilde{\mathcal{G}}}$ coincides with the uniform limiter $\Pi_{L}$ on that cell, or

$$
\left|v\left(x_{L, k^{\prime}}^{+}\right)-v_{L, k^{\prime}, 0}\right| \leq \bar{C}_{L} \varepsilon \text { and }\left|v\left(x_{L, k^{\prime}+1}^{-}\right)-v_{L, k^{\prime}, 0}\right| \leq \bar{C}_{L \varepsilon}
$$

with $\bar{C}_{L}$ according to Lemma 3 .

Proof. By definition of the adaptive limiter, the reference and the adaptive limiter coincide if $(L, k) \in \tilde{\mathcal{G}}$ and $(L, k \pm 1) \in \tilde{\mathcal{G}}$ as well. Otherwise, the adaptive limiter does not change the data. By (B7) and Corollary 2 we have either $c_{L, k^{\prime}}^{ \pm}=1$, and therefore the reference limiter does not change the data as well and the limiters coincide, or

$$
\left|v\left(x_{L, k^{\prime}}^{+}\right)-v_{L, k^{\prime}, 0}\right| \leq \bar{C}_{L} \varepsilon \text { and }\left|v\left(x_{L, k^{\prime}+1}^{-}\right)-v_{L, k^{\prime}, 0}\right| \leq \bar{C}_{L} \varepsilon .
$$

Then by construction of the adaptive limiter and the prediction set we make the following observations:

Lemma 4 (Pointwise error of limiter). Let $\tilde{\mathcal{D}}(\mathbf{w})$ be the prediction set and $\tilde{\mathcal{G}}(\mathbf{w})$ characterize the corresponding adaptive grid determined by the data $\mathbf{w} \in\left(\mathbb{R}^{p}\right)^{N_{L}}$ according to Remark 2 and $w$ the corresponding function. Let $\tilde{u}$ and $u$ be the functions corresponding to $\tilde{\mathbf{u}}:=\Pi_{L, \tilde{\mathcal{G}}(\mathbf{w})} \mathbf{w}$ and $\mathbf{u}:=\Pi_{L} \mathbf{w}$, respectively. Then we can estimate the difference of point evaluations at cell boundaries on the reference grid by

$$
\left|\tilde{u}\left(x_{L, k}^{ \pm}\right)-u\left(x_{L, k}^{ \pm}\right)\right| \leq \bar{C}_{L} \varepsilon \quad \forall k \in\left\{0, \ldots, N_{L}\right\}
$$


with $\bar{C}_{L}$ according to Lemma 3 , where the data at $x_{L, 0}^{-}$and $x_{L, N_{L}}^{+}$are set to 0 , due to the compact support of the solution; see (2.7).

Proof. We confine ourselves to $x_{L, k}^{+}$. By Corollary [3, either the limiters coincide, and then $\tilde{u}\left(x_{L, k}^{+}\right)=u\left(x_{L, k}^{+}\right)$, or we have

$$
\left|w\left(x_{L, k^{\prime}}^{+}\right)-w_{L, k^{\prime}, 0}\right| \leq \bar{C}_{L} \varepsilon .
$$

In the latter case we can represent the limited data as

$$
\begin{aligned}
& \tilde{u}\left(x_{L, k}^{+}\right)-\tilde{u}_{L, k, 0}=w\left(x_{L, k}^{+}\right)-w_{L, k, 0}, \\
& u\left(x_{L, k}^{+}\right)-u_{L, k, 0}=c_{L, k}^{+}\left(w\left(x_{L, k}^{+}\right)-w_{L, k, 0}\right)
\end{aligned}
$$

with some $c_{L, k}^{+} \in[0,1]$. Subtracting (6.8) and (6.9) and using the fact that the limiters do not change the mean values, i.e., $u_{L, k, 0}=\tilde{u}_{L, k, 0}=w_{L, k, 0}$, we obtain

$$
\left|\tilde{u}\left(x_{L, k}^{+}\right)-u\left(x_{L, k}^{+}\right)\right|=\left(1-c_{L, k}^{+}\right)\left|w\left(x_{L, k}^{+}\right)-w_{L, k, 0}\right|,
$$

where we use $\left(1-c_{L, k}^{+}\right) \in[0,1]$. The assertion now follows with (6.7).

6.2. Reliability of limiting: Condition ( $(\underline{\mathrm{L}})$. Since by Lemma 4 the difference in the point values of the adaptive and the reference limiter at the cell interfaces is bounded by the threshold value, this holds true for the mean values when performing one evolution step with the reference DG scheme.

Lemma 5 (Bound on evolution of means). Consider two functions $u, \tilde{u}$ defined by the coefficients $\mathbf{u}, \tilde{\mathbf{u}} \in\left(\mathbb{R}^{p}\right)^{N_{L}}$ with identical mean values, i.e., $\tilde{u}_{L, k, 0}=u_{L, k, 0}$. If the point values at the interfaces of the reference mesh are bounded by the threshold value, i.e., $\left|u\left(x_{L, k}^{ \pm}\right)-\tilde{u}\left(x_{L, k}^{ \pm}\right)\right| \leq \bar{C}_{L} \varepsilon$ with $\bar{C}_{L}$ defined in Lemma 3 , then

$$
\left\|\mathcal{R} \mathcal{E}_{L} \mathbf{u}-\mathcal{R} \mathcal{E}_{L} \tilde{\mathbf{u}}\right\|_{\infty} \leq C \varepsilon
$$

holds, provided that some CFL condition is satisfied. The constant $C$ depends on the point values of $u$ and $\tilde{u}$ at the interfaces.

Proof. For the time evolution of the mean values we conclude by the Lipschitz continuity of the numerical fluxes

$$
\begin{aligned}
& \left\|\mathcal{R E}_{L} \mathbf{u}-\mathcal{R E}_{L} \tilde{\mathbf{u}}\right\|_{\infty} \\
& =\max _{k \in \mathcal{I}_{L}} \mid u_{L, k, 0}-\lambda\left(F\left(u\left(x_{L, k+1}^{-}\right), u\left(x_{L, k+1}^{+}\right)\right)-F\left(u\left(x_{L, k}^{-}\right), u\left(x_{L, k}^{+}\right)\right)\right)- \\
& \tilde{u}_{L, k, 0}+\lambda\left(F\left(\tilde{u}\left(x_{L, k+1}^{-}\right), \tilde{u}\left(x_{L, k+1}^{+}\right)\right)-F\left(\tilde{u}\left(x_{L, k}^{-}\right), \tilde{u}\left(x_{L, k}^{+}\right)\right)\right) \mid \\
& \leq 2 \lambda \max _{k \in \mathcal{I}_{L}}\left|F\left(u\left(x_{L, k}^{-}\right), u\left(x_{L, k}^{+}\right)\right)-F\left(\tilde{u}\left(x_{L, k}^{-}\right), \tilde{u}\left(x_{L, k}^{+}\right)\right)\right| \leq 2 \lambda\left(L_{1} \varepsilon+L_{2} \varepsilon\right),
\end{aligned}
$$

where $L_{1,2}$ are the Lipschitz bounds of the numerical flux function $F$.

Using the estimates in Lemma 4 and Lemma 5 we then conclude

Theorem 2 (Condition $(\underline{\mathrm{L}})$ ). Let $\tilde{\mathcal{D}}(\mathbf{w})$ be the prediction set and $\tilde{\mathcal{G}}(\mathbf{w})$ characterize the corresponding adaptive grid determined by the data $\mathbf{w} \in\left(\mathbb{R}^{p}\right)^{N_{L}}$ according to Remark 2. Then Condition (L) holds, i.e.,

$$
\left\|\mathcal{R}\left(\mathcal{E}_{L} \Pi_{L, \tilde{\mathcal{G}}(\mathbf{w})} b w-\mathcal{E}_{L} \Pi_{L} \mathbf{w}\right)\right\|_{\infty} \leq C_{\Pi} \varepsilon
$$

where $C_{\Pi}$ depends on $\|\mathcal{R} \mathbf{w}\|_{\infty}+\varepsilon+h_{L}^{\alpha}$ due to (2.12) and Lemma 4 , 
6.3. Reliability of evolution: Condition (E). From the construction of the limiter we note that the estimates for the evolution step in Lemma 1 being enforced by the prediction strategy still hold after applying the adaptive limiter, because away from level $L$ the data are not modified.

Lemma 6 (Invariance of estimates after limiting). Let $\tilde{\mathcal{D}}(\mathbf{w})$ be the prediction set determined by the data $\mathbf{w} \in\left(\mathbb{R}^{p}\right)^{N_{L}}$ according to Remark 2 . Then for the function $v_{L}$ corresponding to the limited coefficients $\mathbf{v}:=\Pi_{L, \tilde{\mathcal{G}}(\mathbf{w})} \mathbf{w}$ the estimates (E1), (E2) and (E3) hold for any cell $V_{l, k}$ with $(l, k, i) \notin \tilde{\mathcal{D}}(\mathbf{w})$.

Proof. Let $(l, k, i) \notin \tilde{\mathcal{D}}(\mathbf{w})$. Then there exists a coarse-scale cell $V_{l^{\prime}, k^{\prime}} \supset V_{l, k}$ in the adaptive grid such that $\left(l^{\prime}, k^{\prime}\right) \in \tilde{\mathcal{G}}$ with $l^{\prime}<l \leq L$. According to Case 2 of Algorithm 4 the adaptive limiter does not change the coefficients of this cell. This also holds true for the neighbor cells, because of Case 1. Therefore, neither the coefficients on the cell nor coefficients of its neighbors are modified by the limiter. Hence, the estimates (E1), (E2) and (E3) hold.

We are now ready to verify the reliability condition (4.13) of the evolution step.

Theorem 3 (Growth of non-significant details). We assume that

(1) the flux $f: \mathbb{R} \rightarrow \mathbb{R}$ is sufficiently smooth,

(2) the source function $s: \mathbb{R} \rightarrow \mathbb{R}$ is sufficiently smooth,

(3) the numerical flux $F: \mathbb{R}^{2} \rightarrow \mathbb{R}$ is consistent with the flux $f$ and locally Lipschitz continuous with local Lipschitz constant $L_{F}$,

(4) the temporal discretization $\tau$ is chosen such that a CFL condition holds, i.e., $L_{F} \tau / h_{L} \leq C F L$ holds for a sufficiently small CFL number.

Let $\tilde{\mathcal{D}}(\mathbf{w})$ be the prediction set and $\tilde{\mathcal{G}}(\mathbf{w})$ characterize the corresponding adaptive grid determined by the data $\mathbf{w} \in\left(\mathbb{R}^{p}\right)^{N_{L}}$ according to Remark 2 . Then Lemma 6 implies that the estimates (E1)-(E3) hold for $\overline{\mathbf{w}}:=\Pi_{L, \tilde{\mathcal{G}}(\mathbf{w})} \mathbf{w}$. Consider the evolved data $\mathbf{v}:=\mathcal{E}_{L} \overline{\mathbf{w}}$. Therefore the detail coefficients $d_{l, k, i}$ of $\mathbf{v}$ not contained in the prediction set $\tilde{\mathcal{D}}(\mathbf{w})$ are not significant, i.e.,

$$
\left|d_{l, k, i}\right| \leq C \varepsilon_{l} \quad \forall(l, k, i) \notin \tilde{\mathcal{D}}(\mathbf{w}),
$$

where $C>0$ is a constant depending on the local Lipschitz constant $L_{F}=L_{F}(\mathbf{w})$ of the numerical flux function.

Proof. Let $(l, k, i) \notin \tilde{\mathcal{D}}(\mathbf{w})$. Furthermore, $\bar{w} \in S_{L}^{p}$ and $w \in S_{L}^{p}$ denote the functions corresponding to $\overline{\mathbf{w}}$ and $\mathbf{w}$, respectively. Analogously, $d_{l, k, i}^{\overline{\mathbf{w}}}$ and $d_{l, k, i}^{\mathbf{w}}$ denote the detail coefficients of $\overline{\mathbf{w}}$ and $\mathbf{w}$. Then we note that $\bar{w}=w$ on $V_{l, k}$ and $d_{l, k, i}^{\overline{\mathbf{w}}}=$ $d_{l, k, i}^{\mathbf{w}}=0$ because of ( $(\mathrm{P} 0)$ and the definition of the adaptive limiter. Therefore $\bar{w}=w \in \Pi_{p-1}$ on $V_{l, k}$. Hence, $w$ is differentiable on $V_{l+1,2 k+r}, r=0,1$, and we may apply integration by parts on each subdomain, i.e.,

$$
\begin{aligned}
& \int_{V_{l+1,2 k+r}} f(w(x)) \tilde{\psi}_{l, k, i}^{\prime}(x) d x \\
& =-\int_{V_{l+1,2 k+r}} f(w(x))_{x} \tilde{\psi}_{l, k, i}(x) d x+\left.f(w(y)) \tilde{\psi}_{l, k, i}(y)\right|_{x_{l+1,2 k+r}^{+}} ^{x_{l+2 k+r+1}^{-}} .
\end{aligned}
$$

Since $F_{l, k}^{n}=F_{l+1,2 k}^{n}$ and $F_{l, k+1}^{n}=F_{l+1,2 k+2}^{n}$, see (4.2), as well as $x_{l, k}=x_{l+1,2 k}$ and $x_{l, k+1}=x_{l+1,2 k+2}$, we obtain by rearranging the corresponding terms in (4.7), that

$$
\left|d_{l, k, i}\right| \leq\left|d_{l, k, i}^{\overline{\mathrm{w}}}\right|+\tau\left(\left|T_{1}\right|+\left|T_{2}\right|+\left|T_{3}\right|+\left|T_{4}\right|\right)
$$


with

$$
\begin{aligned}
& T_{1}:=\sum_{r=1}^{2}\left(f\left(w\left(x_{l+1,2 k+r}^{-}\right)\right)-F_{l+1,2 k+r}^{n}\right) \tilde{\psi}_{l, k, i}\left(x_{l+1,2 k+r}^{-}\right), \\
& T_{2}:=\sum_{r=0}^{1}\left(F_{l+1,2 k+r}^{n}-f\left(w\left(x_{l+1,2 k+r}^{+}\right)\right)\right) \tilde{\psi}_{l, k, i}\left(x_{l+1,2 k+r}^{+}\right), \\
& T_{2}:=\int_{V_{l, k}} f(w(x))_{x} \tilde{\psi}_{l, k, i}(x) d x \\
& T_{3}:=\int_{V_{l, k}} s(w(x)) \tilde{\psi}_{l, k, i}(x) d x .
\end{aligned}
$$

We already noted that $d_{l, k, i}^{\overline{\mathrm{w}}}=0$. Therefore the other terms can be estimated with the help of (E1)-(E3). First of all we consider the terms $T_{1}$ and $T_{2}$. Since the numerical flux is assumed to be consistent with the flux $f$, i.e., $F(u, u)=f(u)$ for all $u \in \mathbb{R}$, we first obtain by the local Lipschitz continuity of the numerical flux

$$
\left|f\left(w\left(x_{l+1,2 k+r^{ \pm}}^{ \pm}\right)\right)-F_{l+1,2 k+r^{ \pm}}^{n}\right| \leq L_{F}\left|w\left(x_{l+1,2 k+r^{ \pm}}^{-}\right)-w\left(x_{l+1,2 k+r^{ \pm}}^{+}\right)\right|
$$

for $r^{+}=0,1$ and $r^{-}=1,2$. Note that the Lipschitz constant depends on the data, i.e., $L_{F}=L_{F}(\mathbf{w})$.

Since the dual functions $\tilde{\psi}_{l, k, i}$ are piecewise polynomials and the $L^{1}$-normalized (3.6) we may bound them by

$$
\left|\tilde{\psi}_{l, k, i}(x)\right| \lesssim 2^{l} \quad \forall x \in \mathbb{R} .
$$

Moreover, $w$ is continuous inside the cell $V_{l, k}$. Therefore, only the differences at the cell interfaces, i.e., $x=x_{l, k}$ and $x=x_{l, k+1}$, give a non-trivial contribution. Hence we can estimate the sum $T_{1}+T_{2}$ by

$$
\left|T_{1}\right|+\left|T_{2}\right| \lesssim 2^{l}\left(\left|w\left(x_{l, k}^{+}\right)-w\left(x_{l, k}^{-}\right)\right|+\left|w\left(x_{l, k+1}^{+}\right)-w\left(x_{l, k+1}^{-}\right)\right|\right) .
$$

Together with (E1) we finally obtain

$$
\left|T_{1}\right|+\left|T_{2}\right| \lesssim 2^{l} \varepsilon .
$$

In order to estimate the third term $T_{3}$ we will use the vanishing moments of the wavelets. First of all, we write the derivative of the flux in a Taylor expansion

$$
\frac{d}{d x} f(w(x))=\sum_{r=0}^{p-1} \frac{(x-\hat{x})^{r}}{r !} \frac{d^{r+1}}{d x^{r+1}} f(w(\hat{x}))+R_{p-1}(x),
$$

where the reminder is determined by

$$
R_{p-1}(x)=\frac{1}{p !}(x-\hat{x})^{p} \frac{d^{p+1}}{d x^{p+1}} f(w(\xi)), \quad \xi \in[x, \hat{x}] .
$$

Then we estimate

$$
\begin{aligned}
\left|T_{3}\right| & =\left|\int_{V_{l, k}}(f(w(x)))_{x} \tilde{\psi}_{l, k, i}(x) d x\right|=\left|\int_{V_{l, k}} R_{p-1}(x) \tilde{\psi}_{l, k, i}(x) d x\right| \\
& \leq\left\|R_{p-1}\right\|_{L^{\infty}\left(V_{l, k}\right)}\left\|\tilde{\psi}_{l, k, i}\right\|_{L^{1}\left(V_{l, k}\right)} \lesssim \frac{\left(h_{l} / 2\right)^{p}}{p !}\left\|\frac{d^{p+1}}{d x^{p+1}} f(w)\right\|_{L^{\infty}\left(V_{l, k}\right)} \lesssim 2^{l} \varepsilon,
\end{aligned}
$$


where we choose $\hat{x}=\hat{x}_{l, k}$. Note that the dual multiwavelets $\tilde{\psi}_{l, k, i}$ have at least $p$ vanishing moments, i.e., $M_{p, i} \geq p$ in (3.7), and are $L^{1}$-normalized according to (3.6). The last inequality is enforced by (E2).

Finally we have to estimate the fourth term. This is done as before for the third term, using the smoothness of the source function $s$, the vanishing moments and estimate (E3):

$$
\left|T_{4}\right|=\left|\int_{V_{l, k}} s(w(x)) \tilde{\psi}_{l, k, i}(x) d x\right| \lesssim \frac{\left(h_{l} / 2\right)^{p}}{p !}\left\|\frac{d^{p}}{d x^{p}} s(w(\cdot))\right\|_{L^{\infty}\left(V_{l, k}\right)} \lesssim 2^{l} \varepsilon .
$$

Since a CFL condition is assumed to hold with respect to level $L$, i.e., $\tau \sim 2^{-L}$, the assertion follows by (6.16), (6.17) and (6.18).

Using the above theorem, we can now verify condition (E):

Theorem 4 (Condition $(\mathrm{E})$ ). Let $\tilde{\mathcal{D}}(\mathbf{w})$ be the prediction set and $\tilde{\mathcal{G}}(\mathbf{w})$ characterize the corresponding adaptive grid determined by the data $\mathbf{w} \in\left(\mathbb{R}^{p}\right)^{N_{L}}$ according to Remark 2 and $\mathbf{u}=\Pi_{L, \tilde{\mathcal{G}}(\mathbf{w})} \mathbf{w}$. If the flux and source integral are performed exactly, then Condition (E) holds, i.e.,

$$
\left\|\mathcal{R}\left(\mathcal{E}_{L, \tilde{\mathcal{G}}(\mathbf{w})} \mathbf{u}-\mathcal{E}_{L} \mathbf{u}\right)\right\|_{\infty} \leq C_{\mathcal{E}} \varepsilon
$$

Proof. We note that by Lemma 6 the estimates (E1)-(E3) hold for $\mathbf{u}$. In order to show (E), we have to investigate the difference of $\overline{\mathbf{v}}:=\mathcal{E}_{L, \tilde{\mathcal{G}}(\mathbf{w})} \mathbf{u}$ and $\mathbf{v}:=\mathcal{E}_{L} \mathbf{u}$. For this purpose we apply the multi-scale transformation (3.14) to $\overline{\mathbf{v}}$ and $\mathbf{v}$, respectively. The resulting multi-scale coefficients are denoted by $\bar{v}_{0, k, i}, \bar{d}_{l, k, i}$ and $v_{0, k, i}, d_{l, k, i}$, respectively. Since the evolution operators $\mathcal{E}_{L, \tilde{\mathcal{G}}(\mathbf{w})}$ and $\mathcal{E}_{L}$ act on the same data and the integrations are performed exactly, the evolution equations (4.6) and (4.7) give the same update for the coarse-scale coefficients and the significant detail coefficients, i.e., $\bar{v}_{0, k, i}=v_{0, k, i}$ and $\bar{d}_{l, k, i}=d_{l, k, i},(l, k, i) \in \tilde{\mathcal{D}}(\mathbf{w})$. There is only a difference for the details not in the prediction set. According to the adaptive MR-DG scheme no evolution is performed for these coefficients, i.e., $\bar{d}_{l, k, i}=0$, $(l, k, i) \notin \tilde{\mathcal{D}}(\mathbf{w})$, whereas these are updated for the reference DG scheme. By Theorem 3 applied to $\mathbf{u}$ we have $\left|d_{l, k, i}\right| \leq C \varepsilon_{l}$. To estimate the difference $\overline{\mathbf{v}}-\mathbf{v}$ we thus may proceed as in the proof of the approximation error; see Proposition 2 .

\subsection{Proof of Theorem 1 .}

Proof. By Theorems 2 and 4 the conditions (E) and (L) are satisfied. Furthermore Proposition 2 ensures that the approximation error introduced by the threshold operator is bounded by (3.22). Then we may conclude for any data $\mathbf{w} \in\left(\mathbb{R}^{p}\right)^{N_{L}}$ :

$$
\begin{aligned}
\left\|\mathcal{R}\left(\mathcal{H}_{\varepsilon} \mathbf{w}-\mathcal{H} \mathbf{w}\right)\right\|_{\infty}=\| & \mathcal{R}\left(\mathcal{A}_{\varepsilon} \mathcal{E}_{L, \tilde{\mathcal{G}}(\mathbf{w})} \Pi_{L, \tilde{\mathcal{G}}(\mathbf{w})} \mathbf{w}-\mathcal{E}_{L} \Pi_{L} \mathbf{w}\right) \|_{\infty} \\
\leq \| & \mathcal{R}\left(\mathcal{A}_{\varepsilon} \mathcal{E}_{L, \tilde{\mathcal{G}}(\mathbf{w})} \Pi_{L, \tilde{\mathcal{G}}(\mathbf{w})} \mathbf{w}-\mathcal{E}_{L, \tilde{\mathcal{G}}(\mathbf{w})} \Pi_{L, \tilde{\mathcal{G}}(\mathbf{w})} \mathbf{w}\right) \|_{\infty} \\
& +\left\|\mathcal{R}\left(\mathcal{E}_{L, \tilde{\mathcal{G}}(\mathbf{w})} \Pi_{L, \tilde{\mathcal{G}}(\mathbf{w})} \mathbf{w}-\mathcal{E}_{L} \Pi_{L, \tilde{\mathcal{G}}(\mathbf{w})} \mathbf{w}\right)\right\|_{\infty} \\
& \quad+\left\|\mathcal{R}\left(\mathcal{E}_{L} \Pi_{L, \tilde{\mathcal{G}}(\mathbf{w})} \mathbf{w}-\mathcal{E}_{L} \Pi_{L} \mathbf{w}\right)\right\|_{\infty} \\
\leq & C_{t h r} \varepsilon+C_{\mathcal{E}} \varepsilon+C_{\Pi} \varepsilon=C \varepsilon
\end{aligned}
$$

where $\tilde{\mathcal{D}}(\mathbf{w})$ is the prediction set and $\tilde{\mathcal{G}}(\mathbf{w})$ characterizes the corresponding adaptive grid determined by $\mathbf{w}$ according to Remark 2, We note that the constants $C_{\mathcal{E}}$ and $C_{\Pi}$ depend on the local Lipschitz constants of the numerical flux function, and 
therefore the constants depend on the data $\Pi_{L, \tilde{\mathcal{G}}(\mathbf{w})} \mathbf{w}$ and $\Pi_{L} \mathbf{w}$ at the interfaces. Both of these terms can be estimated by $\|\mathcal{R} \mathbf{w}\|_{\infty}+C_{\alpha} h_{L}^{\alpha}+\varepsilon$, due to Lemma 4 and (2.12). Since $h_{L}$ and $\varepsilon$ are assumed to be bounded from above, we conclude that condition (5.6) holds for all $\mathbf{w} \in\left(\mathbb{R}^{p}\right)^{N_{L}}$ with $\|\mathcal{R} \mathbf{w}\|_{\infty} \leq C_{\infty}$, where $C_{\infty}$ is defined in Proposition 3 Then, in particular, $C_{1}:=C_{t h r}+C_{\mathcal{E}}+C_{\Pi}$ is independent of $\varepsilon$ and $h_{L}$. Furthermore, we have by (3.22) a bound for the error in the initial data, i.e., condition (5.7) holds with $C_{2}$ determined by Proposition 2 Hence Assumption 1 holds and we may apply Proposition 3.

\section{Numerical RESULTS}

In order to verify the analytical results and the efficiency of the adaptive MR-DG scheme, numerous numerical computations have been performed for scalar as well as systems of conservation laws; cf. [42, 29]. Exemplarily we present here results for the inhomogeneous inviscid Burgers' equation with flux $f(u)=0.5 u^{2}$ and source function $s(u)=u(u-0.5)(u-1)$. For initial data we choose $u_{0}(x)=\sin (2 \pi x)$ in $\Omega=[0,1]$. The boundaries are assumed to be periodic. Thus, a shock is developing at some time $t>0$ that is moving at negative speed. Note that at the boundaries the characteristic speeds are zero, i.e., the solution does not change there.

Settings. The computational domain is discretized by $N_{0}=5$ cells on the coarsest level, i.e., $h_{0}=0.2$. Hence the resolution for higher refinement levels is $N_{l}=2^{l} N_{0}$ and $h_{l}=2^{-l} h_{0}$. The number of refinement levels is $L=9$. For the time discretization, we use the $\operatorname{SSP}-\mathrm{RK}(3,3)$ scheme; cf. 43 . We have to respect a CFL condition of $C F L=0.1$ to ensure both linear $L^{2}$ - and non-linear $T V D$ stability; therefore we choose $\tau_{0}=0.005$. Since we perform global time stepping, the CFL condition has to hold for the smallest cells corresponding to the highest refinement level $L$, i.e., $\tau=2^{-L} \tau_{0}=10^{-5}$. The final integration time is $T=0.24$. We choose piecewise cubic polynomials for the discretization space $S_{h}^{p}$, i.e. $p=4$. The reference DG scheme (2.6) is determined by the Engquist-Osher flux, and the integrals in the integral terms (2.9) are computed by a 5-point Gaussian quadrature rule of order 9, i.e., exact integration. Limiting is performed using (2.10), where we do not incorporate the entropy bound (2.12) because we consider a convex flux; see [39].

Grid refinement. The results of the adaptive DG scheme are presented in Figure 4 for varying threshold values $\varepsilon=10^{-1 / 2}, \ldots, 10^{-3}$. For each computation we plot the adaptive solution (top), the adaptive grid after hard thresholding (middle) characterized by the set $\mathcal{D}$ corresponding to the significant details and the predicted grid corresponding to the prediction set $\tilde{\mathcal{D}}$ (bottom), which was used to calculate that solution. There is hardly any difference visible between the solution of the adaptive DG scheme and the reference DG scheme performed on the uniform mesh corresponding to the highest refinement level $L=9$. However, there are differences in the locally refined grids. These are represented by the position of the local cells in the grid hierarchy. To each cell pair $\left(V_{l+1,2 k}, V_{l+1,2 k+1}\right)$ four grey scales are shown for the absolute value of the multi-scale coefficients $\left|d_{l, k, i}\right|, i=0, \ldots, 3$, where white corresponds to 0 and black to the maximal value on level $l$. The magnitude of the scales is given as labels on the right axis. Typically the absolute value of these coefficients in smooth regions decreases with increasing order $i$, due to the $i+p$ vanishing moments. We note that the largest values occur at the discontinuity. Therefore the grid is locally refined near to its position at $x \approx 0.41$. Away from 



Figure 2. Variation of $\# \tilde{\mathcal{D}} / \# \mathcal{D}$ (left) and error of adaptive solution (right) with $L=9$ for different threshold values $\varepsilon$.

this isolated point, the discretization is coarse because of the local smoothness of the solution. We note that the adaptive grid is inflated due to the prediction.

Perturbation error versus discretization error. In order to investigate the discretization errors of the adaptive and the reference scheme, we need to determine the entropy solution. Since this solution is not explicitly available for the problem at hand, we use a computation with the reference DG scheme on a uniformly refined grid with four additional refinement levels for the "exact" solution as is usually done. Then the discretization error $\hat{\eta}_{L}=\hat{u}-\hat{v}$ of the reference scheme for $L=9$ is $\left\|\hat{\eta}_{L}\right\|_{L^{1}([0,1])}=7.1 \times 10^{-4}$. For varying threshold values we then plot in Figure 2 (right) the discretization error of the adaptive scheme $\hat{u}-\hat{v}_{\varepsilon}$ and the perturbation error $\hat{e}:=\hat{v}-\hat{v}_{\varepsilon}$. Obviously, the perturbation error is decreasing with smaller threshold values. In particular, it tends to zero for $\varepsilon \rightarrow 0^{+}$, i.e., since in the limit the adaptive scheme and the reference scheme coincide for fixed $L$. Therefore we do not gain accuracy when choosing a very small threshold value, because the discretization error of the adaptive scheme is bounded by the discretization error of the reference scheme; see the horizontal line in Figure 2 (right).

Optimal choice of threshold value. For a fixed refinement level $L$ the ideal strategy presented in Section 5 suggests that the optimal threshold value $\varepsilon_{\text {opt }}$ should be chosen such that the discretization error $\hat{\eta}_{L}=\hat{u}-\hat{v}_{L}$ of the reference scheme and the perturbation error $\hat{e}_{L}=\hat{v}_{L}-\hat{v}_{L, \varepsilon}$ are balanced. As we can depict from Figure 2 (right) an optimal choice would be $\varepsilon_{o p t} \sim 10^{-1.75}$, since for $\varepsilon>\varepsilon_{\text {opt }}$ the perturbation error dominates, whereas for $\varepsilon<\varepsilon_{o p t}$ the discretization error dominates.

In practice, we do not know $\varepsilon_{o p t}$ explicitly. In case of scalar, one-dimensional problems, where according to Sections [5 and [6 we have some analysis available, Corollary 1] suggests the choice $\varepsilon \sim h_{L}^{1+\beta}$. According to 12] the discretization error of the reference scheme is of order $h_{L}^{\beta}$ with $\beta=\min \{1, \alpha\} / 2$, and $\alpha$ corresponds to 

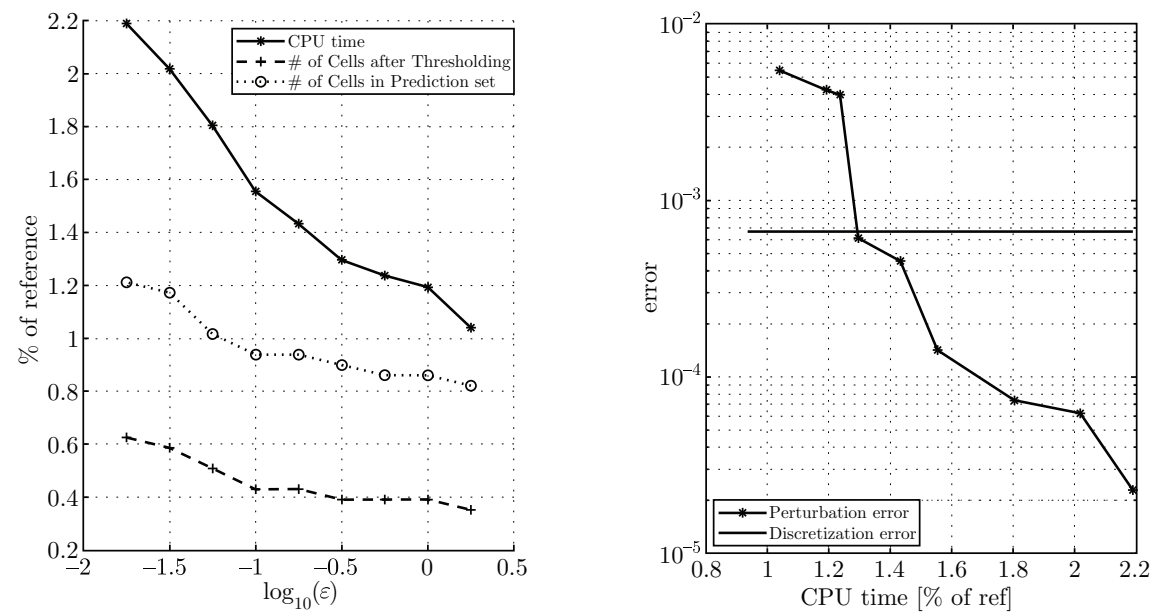

FiguRE 3. Number of cells and CPU time w.r.t. reference solution (left) and comparison of CPU time and error of adaptive scheme.

the entropy bound (2.10b) in the limiter. Then we compute for our configuration $h_{L}^{1+\beta} \sim 7.7 \times 10^{-6}$, where we put $\alpha \geq 1$ and $C_{\alpha}=\infty$ and $h_{L}=0.2 \times 2^{-9}$. Obviously, this value is by several orders smaller than $\varepsilon_{o p t}$ and, hence, too pessimistic. Since Corollary 11 relies on a priori error estimates of the discretization error and the perturbation error, it is a worst case estimate, i.e., the threshold error is assumed to accumulate over all time steps and, hence, we are losing one order of magnitude to compensate for the accumulation. As a rule of thumb we therefore recommend to use $\varepsilon \sim h_{L}^{\beta}$ in practice. In fact, for our computations we obtain $h_{L}^{\beta} \approx 10^{-1.7}$ that fits much better to $\varepsilon_{\text {opt }}$.

Efficiency of the prediction step. The performance of the adaptive scheme essentially relies on the cardinality of the prediction set $\tilde{\mathcal{D}}$ or, equivalently, on the number of cells in the adaptive grid. Since by the prediction step we inflate the set $\mathcal{D}$ of significant detail coefficients, it is crucial that the prediction step works efficiently, i.e., the ratio $\# \tilde{\mathcal{D}} / \# \mathcal{D} \geq 1$ should not be too large. In Figure 2 (left) the ratio of the prediction set $\tilde{\mathcal{D}}$ and the set $\mathcal{D}$ of significant details is plotted versus the threshold value. It ranges between 1.9 and 2.4 for varying threshold values and the fixed number of refinement levels. This indicates a reasonably efficient prediction strategy. Note that the absolute numbers $\# \tilde{\mathcal{D}}$ and $\# \mathcal{D}$ are negligibly small in comparison to the uniform reference mesh.

Computational costs. First we discuss the size of the adaptive grids that determine the memory requirements; see Figure 3 (left). We observe that with decreasing threshold value the number of cells is increasing and, thus, results in higher CPU times. Since the ultimate goal is to save computational costs and to preserve the accuracy of the reference solution, we plot in Figure 3 (right) the perturbation error for varying threshold values versus the CPU time. We note that the CPU time is at most $2.5 \%$ of the computation with the reference scheme. For the optimal threshold value $\varepsilon_{o p t}$ it is only about $2 \%$, i.e., we gain a factor 50 where the perturbation error balances the discretization error. Since in practice we only 

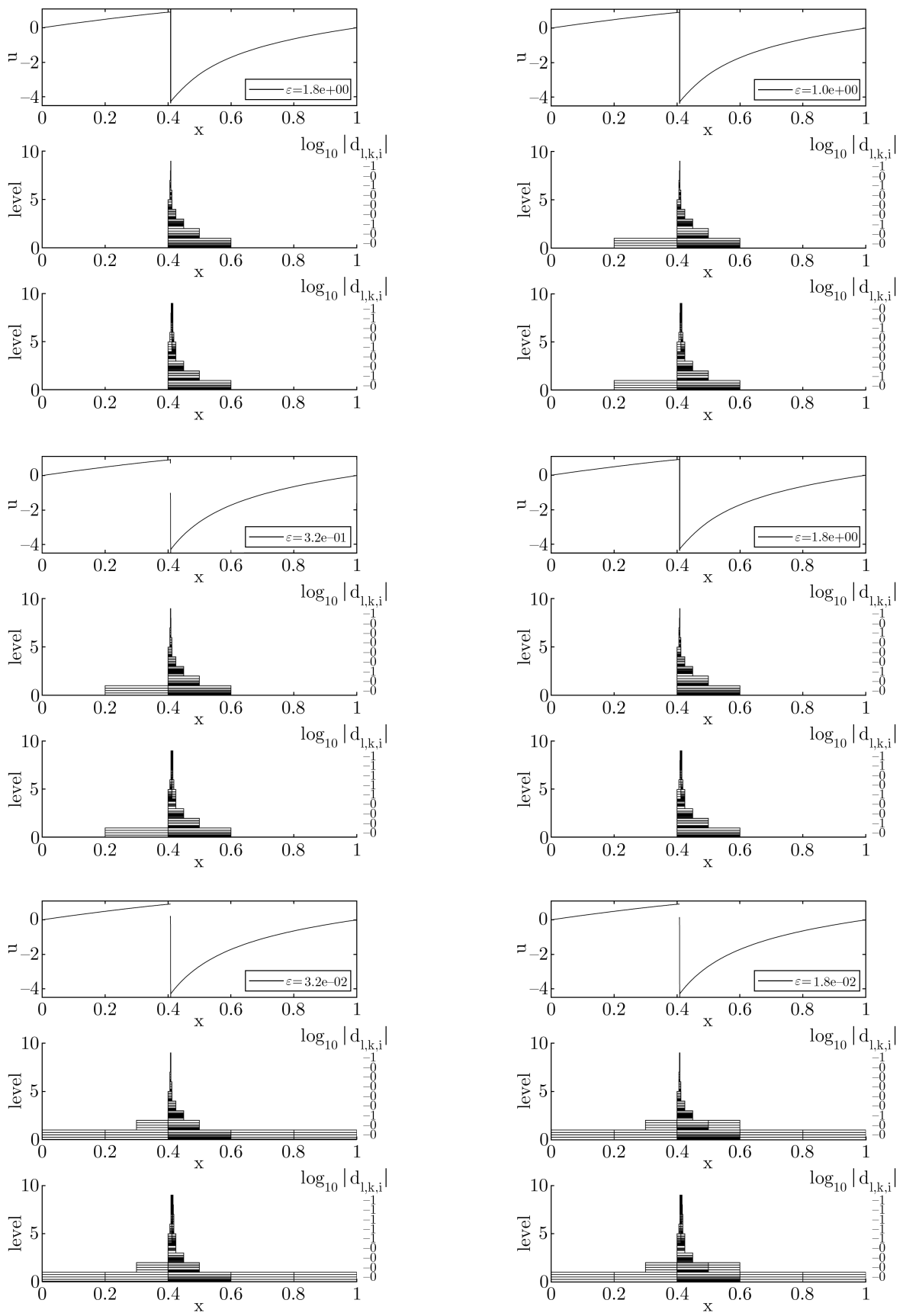

Figure 4. Results of the adaptive MR-DG scheme at time $T=$ 0.24 using different threshold values $\varepsilon$. For each value the adaptive solution (top), the adaptive grid after hard thresholding (middle) and the predicted grid corresponding to the prediction set (bottom) are shown in each frame. 
can provide some heuristic law for $\varepsilon_{o p t}$, it is important to note that we are far from significantly losing efficiency when our guess is too pessimistic.

\section{ACKNowledGments}

The authors greatly appreciate the reviewers' numerous remarks and suggestions that helped to significantly improve the presentation of this work.

\section{REFERENCES}

[1] S. Adjerid, K.D. Devine, J.E. Flaherty, and L. Krivodonova. A posteriori error estimation for discontinuous Galerkin solutions of hyperbolic problems. Comput. Methods Appl. Mech. Eng., 191:1097-1112, 2002. MR 1877682(2002k:65137)

[2] B. Alpert. A class of bases in $l^{2}$ for the sparse representation of integral operators. SIAM J. Math. Anal., 24:246-262, 1993. MR1199538(93k:65104)

[3] B. Alpert, G. Beylkin, D. Gines, and L. Vozovoi. Adaptive solution of partial differential equation in multiwavelet bases. J. Comp. Phys., 182:149-190, 2002. MR1936805(2003i:65083)

[4] R.M. Beam and R.F. Warming. Multiresolution analysis and supercompact multiwavelets. SIAM J. Sci. Comput., 22(4):1238-1268, 2000. MR1787295 (2001f:42056)

[5] K.S. Bey and J.T. Oden. hp-version discontinuous Galerkin methods for hyperbolic conservation laws. Comput. Methods Appl. Mech. Eng., 133(3-4):259-286, 1996. MR1399638 (97g:65225)

[6] F. Bramkamp, Ph. Lamby, and S. Müller. An adaptive multiscale finite volume solver for unsteady and steady state flow computations. J. Comp. Phys., 197(2):460-490, 2004. MR2063903(2005a:76106)

[7] K. Brix, R. Massjung, and A. Voss. Refinement and connectivity algorithms for adaptive discontinuous Galerkin methods. SIAM J. Sci. Comput., 33(1):66-101, 2011. MR2783187 (2012g:65252)

[8] B. Cockburn. Quasimonotone schemes for scalar conservation laws: Part I. SIAM J. Numer. Anal., 26(6):1325-1341, 1989. MR.1025091 (91b:65106)

[9] B. Cockburn, S. Hou, and C.-W. Shu. The Runge-Kutta local projection discontinuous Galerkin finite element method for conservation laws. IV: The multidimensional case. Math. Comp., 54(190):545-581, 1990. MR.1010597(90k:65162)

[10] B. Cockburn, G.E. Karniadakis, and C.-W. Shu. The development of discontinuous Galerkin methods. Cockburn, Bernardo (ed.) et al., Discontinuous Galerkin methods. Theory, computation and applications. 1st international symposium on DGM, Newport, RI, USA, May 24-26, 1999. Berlin: Springer. Lect. Notes Comput. Sci. Eng. 11, 3-50 (2000). MR1842161 (2002e:65002)

[11] B. Cockburn, S.-Y. Lin, and C.-W. Shu. TVB Runge-Kutta local projection discontinuous Galerkin finite element method for conservation laws. III: One-dimensional systems. J. Comput. Phys., 84(1):90-113, 1989. MR1015355(90k:65161)

[12] B. Cockburn and C.-W. Shu. TVB Runge-Kutta local projection discontinuous Galerkin finite element method for conservation laws II: General framework. Math. Comp., 52(186):411-435, 1989. MR983311 (90k:65160)

[13] B. Cockburn and C.-W. Shu. The Runge-Kutta local projection $p^{1}$-discontinuous-Galerkin finite element method for scalar conservation laws. RAIRO Modélisation Math. Anal. Numér., 25(3):337-361, 1991. MR 1103092 (92e:65128)

[14] B. Cockburn and C.-W. Shu. The Runge-Kutta discontinuous Galerkin method for conservation laws. V: Multidimensional systems. J. Comput. Phys., 141(2):199-224, 1998. MR.1619652 (99c:65181)

[15] B. Cockburn and C.-W. Shu. Runge-Kutta discontinuous Galerkin methods for convectiondominated problems. J. Sci. Computing, 16(3):173-261, 2001. MR.1873283 (2002i:65099)

[16] A. Cohen. Numerical analysis of wavelet methods, volume 32 of Studies in Mathematics and its Applications. North-Holland Publishing Co., Amsterdam, 2003. MR 1990555 (2004c:65178)

[17] A. Cohen, I. Daubechies, and J. Feauveau. Biorthogonal bases of compactly supported wavelets. Comm. Pure Appl. Math., 45:485-560, 1992. MR.1162365 (93e:42044) 
[18] A. Cohen, S.M. Kaber, S. Müller, and M. Postel. Fully adaptive multiresolution finite volume schemes for conservation laws. Math. Comp., 72(241):183-225, 2003. MR.1933818 (2003k:65109)

[19] F. Coquel, Y. Maday, S. Müller, M. Postel, and Q.H. Tran. New trends in multiresolution and adaptive methods for convection-dominated problems. ESAIM, Proc., 29:1-7, 2009. MR.2766462 (2011i:65005)

[20] W. Dahmen, B. Gottschlich-Müller, and S. Müller. Multiresolution schemes for conservation laws. Numer. Math., 88(3):399-443, 2000. MR1835465(2002e:65116)

[21] A. Dedner, C. Makridakis, and M. Ohlberger. Error control for a class of Runge Kutta discontinuous Galerkin methods for nonlinear conservation laws. SIAM J. Numer. Anal., 45:514-538, 2007. MR2300285 (2008d:65109)

[22] E. Godlewski and P.-A. Raviart. Hyperbolic systems of conservation laws, volume 3/4 of Mathématiques \& Applications (Paris) [Mathematics and Applications]. Ellipses, Paris, 1991. MR 1304494 (95i:65146)

[23] S. Gottlieb, C.-W. Shu, and E. Tadmor. Strong stability preserving high-order time discretization methods. SIAM Review, 43(1):89-112, 2001. MR1854647 (2002f:65132)

[24] A. Harten. Multiresolution representation of data: A general framework. SIAM J. Numer. Anal., 33(3):1205-1256, 1996. MR 1393910(97h:65078)

[25] R. Hartmann and P. Houston. Adaptive discontinuous Galerkin finite element methods for nonlinear hyperbolic conservation laws. SIAM J. Sci. Comput., 24:979-1004, 2002. MR.1950521 (2004b:65149)

[26] R. Hartmann and P. Houston. Adaptive discontinuous Galerkin finite element methods for the compressible Euler equations. J. Comp. Phys., 183:508-532, 2002. MR1947780|(2003k:76084)

[27] P. Houston, B. Senior, and E. Süli. $h p$-discontinuous Galerkin finite element methods for hyperbolic problems: Error analysis and adaptivity. Int. J. Numer. Methods Fluids, 40(12):153-169, 2002. MR.1928323 (2003g:65144)

[28] N. Hovhannisyan and S. Müller. On the stability of fully adaptive multiscale schemes for conservation laws using approximate flux and source reconstruction strategies. IMA Journal of Numerical Analysis, 30:1256-1295, 2010. MR2727824 (2012b:65121)

[29] F. Iacono, G. May, S. Müller, and R. Schäfer. A high-order discontinuous Galerkin discretization with multiwavelet-based grid adaptation for compressible flows. AICES Preprint AICES-2011/08-1, RWTH Aachen, 2011.

[30] F. Keinert. Wavelets and multiwavelets. Studies in Advanced Mathematics. Chapman \& Hall/CRC, Boca Raton, FL, 2004. MR2035222 (2005b:42001)

[31] D. Kröner. Numerical schemes for conservation laws. Chichester: Wiley; Stuttgart: Teubner, 1997. MR1437144(98b:65003)

[32] S.N. Kruzhkov. First order quasilinear equations in several independent variables. Math. USSR Sbornik, 10:217-243, 1970.

[33] R.J. LeVeque. Finite volume methods for hyperbolic problems. Cambridge Texts in Applied Mathematics. Cambridge University Press, Cambridge, 2002. MR1925043 (2003h:65001)

[34] S. G. Mallat. A theory for multiresolution signal decomposition: The wavelet representation. IEEE Trans. Pattern Anal. Machine Intell., 11(7):674-693, 1989.

[35] S. Müller. Adaptive multiscale schemes for conservation laws, volume 27 of Lecture Notes in Computational Science and Engineering. Springer-Verlag, Berlin, 2003. MR,1952371 (2004b:76102)

[36] S. Müller. Multiresolution schemes for conservation laws. DeVore, Ronald (ed.) et al., Multiscale, nonlinear and adaptive approximation. Dedicated to Wolfgang Dahmen on the occasion of his 60th birthday. Springer, Berlin. 379-4080, 2009. MR2648379 (2011i:65142)

[37] S. Müller and A. Voss. A Manual for the Template Class Library igpm_t_lib. IGPM-Report 197, RWTH Aachen, 2000.

[38] O.A. Oleinik. Discontinuous solutions of nonlinear differential equations. Amer. Math. Soc. Transl. Ser. 2, 26:95-172, 1963. MR0151737 (27:1721)

[39] S. Osher. Convergence of generalized MUSCL schemes. SIAM J. Numer. Anal., 22(5):947961, 1985. MR799122 (87b:65147)

[40] W.H. Reed and T.R. Hill. Triangular mesh methods for neutron transport equation. Technical Report LA-UR-73-479, Los Alamos Scientific Laboratory, Los Alamos, NM, 1973. 
[41] J.-F. Remacle, J.E. Flaherty, and M.S. Shephard. An adaptive discontinuous Galerkin technique with an orthogonal basis applied to compressible flow problems. SIAM Review, 45(1):53-72, 2003. MR1981254 (2004k:65176)

[42] R. Schäfer. Adaptive multiresolution discontinuous Galerkin schemes for conservation laws. Ph.D. thesis, RWTH Aachen, 2011.

[43] C.-W. Shu and S. Osher. Efficient implementation of essentially non-oscillatory shockcapturing scheme. J. Comp. Physics, 77:439-471, 1988. MR954915 (89g:65113)

[44] V. Strela. Multiwavelets: Theory and applications. Ph.D. thesis, Massachusetts Institute of Technology, 1996. MR2716668

[45] T. Tang and Z.-H. Teng. Error bounds for fractional step methods for conservation laws with source terms. SIAM J. Numer. Anal., 32(1):110-127, 1995. MR1313707 (95m:65155)

[46] L. Wang and D.J. Mavriplis. Adjoint-based $h p$ adaptive discontinuous Galerkin methods for the 2d compressible Euler equations. J. Comp. Phys., 228(20):7643-7661, 2009. MR2561836

[47] R.F. Warming and R.M. Beam. Discrete multiresolution analysis using Hermite interpolation biorthogonal multiwavelets. SIAM J. Sci. Comput., 22(4):1269-1317, 2000. MR.1787296 (2001e:42055)

Faculty of Informatics and Applied Mathematics, Alex Manoogian 1, Yerevan 0025, ARMEnia

E-mail address: alnune03@yahoo.com

Institut für Geometrie und Praktische Mathematik, RWTH Aachen University, TemPlergraben 55, 52056 Aachen, Germany

E-mail address: mueller@igpm.rwth-aachen.de

Institut für Geometrie und Praktische Mathematik, RWth Aachen University, TemPlergraben 55, 52056 Aachen, Germany

E-mail address: schaefer@igpm.rwth-aachen.de 